\title{
Temporal and Spatial Tradeoffs in Power System Modeling with Assumptions about Storage: An Application of the POWER Model
}

\author{
Bethany A. Frew a," and Mark Z. Jacobson ${ }^{\mathrm{a}}$ \\ ${ }^{a}$ Civil and Environmental Engineering, Stanford University, Stanford, CA
}

\begin{abstract}
As the number and complexity of power system planning models grows, understanding the impact of modeling choices on accuracy and computational requirements becomes increasingly important. This study examines empirically various temporal and spatial tradeoffs using the POWER planning model for scenarios of a highly renewable US system. First, the common temporal simplification of using a representative subset of hours from a full year of available hours is justified using a reduced form model. Accuracy losses are generally $\leq 6 \%$, but storage is sensitive to the associated model modifications, highlighting the need for proper storage balancing constraints. Cost tradeoffs of various temporal and spatial adjustments are then quantified: four temporal resolutions (1- to 8-hour-average time blocks); various representative day subset sizes ( 1 week to 6 months); two spatial resolutions of site-by-site versus uniform fractional buildout across all solar and wind sites; and multiple spatial extents, ranging from California to the contiguous US. Most tradeoffs yield $<15 \%$ cost differences, with the effect of geographic aggregation across increasing spatial extents producing the largest cost reduction of $14 \%$ and $42 \%$ for the western and contiguous US, respectively. These results can help power system modelers determine the most appropriate temporal and spatial treatment for their application.
\end{abstract}

Keywords: renewable energy; linear programming; computational requirements; model accuracy; aggregation; energy system analysis

\footnotetext{
* Corresponding author. Tel.: +1 650721 2650; fax: +1 6507237058
}

E-mail address: bethany.frew@alumni.stanford.edu 


\section{Introduction}

As the US electricity sector transforms to meet regulatory and reliability requirements in an aging and increasingly renewable system, numerous optimization studies are being conducted to explore the economic and power system impacts under different generator and transmission scenarios. These studies span a range of spatial scales, from regional, state, and balancing areas, e.g., PJM using the RREEOM model [1] and the Western US using the SWITCH model [2], to country-wide analyses, e.g., contiguous US using the ReEDS model [3], US-REGEN model[4], NEWS model [5], and POWER model [6]. Many of these studies utilize a specific multi-decade capacity expansion model or shorter-term planning model. Table 1 summarizes the relevant features of several US national models (POWER, ReEDS, US-REGEN, NEWS, NEMS, ReNOT) and large regional models (SWITCH, RREEOM). Each of these models deterministically optimizes for the least-cost system. A review of these model can be found in Section 4.1 of [7]; a broader review of optimization, simulation, and equilibrium capacity expansion models is provided in [8].

Table 1 High level comparison of selected US power system planning models. TS=time-slice. $R \& C=$ renewable and conventional. WECC=Western Electricity Coordinating Council. FERC=Federal Energy Regulatory Commission.

\begin{tabular}{|c|c|c|c|c|c|c|}
\hline Model & $\begin{array}{c}\text { Spatial } \\
\text { Resolution/Extent }\end{array}$ & $\begin{array}{c}\text { Temporal } \\
\text { Resolution/Extent }\end{array}$ & $\begin{array}{c}\text { Generator } \\
\text { Technologies }\end{array}$ & Storage? & Transmission? & Source(s) \\
\hline $\begin{array}{l}\text { POWER } \\
\text { (Stanford) }\end{array}$ & $\begin{array}{l}10 \text { FERC regions; } \\
\sim 6000 \text { wind and } \\
\sim 1400 \text { PV and CSP } \\
\text { sites; US }\end{array}$ & $\begin{array}{l}\text { Hourly with 14+ } \\
\text { TS/yr }\end{array}$ & Various $R \& C$ & Yes & Inter-regional & {$[6,7]$} \\
\hline $\begin{array}{l}\text { ReEDS } \\
\text { (NREL) }\end{array}$ & $\begin{array}{l}134 \text { load and PV } \\
\text { resource regions; } \\
356 \text { wind and CSP } \\
\text { resource regions; } \\
\text { US }\end{array}$ & $\begin{array}{l}\text { Hourly with } 17 \text { TS } \\
\text { blocks/yr }\end{array}$ & Various $R \& C$ & Yes & $\begin{array}{l}\text { Inter- \& intra- } \\
\text { regional }\end{array}$ & [9] \\
\hline $\begin{array}{l}\text { SWITCH (UC } \\
\text { Berkeley) }\end{array}$ & $\begin{array}{l}50 \text { load regions; } \\
\sim 3000 \text { sites each } \\
\text { wind, PV, and CSP; } \\
\text { WECC }\end{array}$ & $\begin{array}{l}\text { Hourly with } 144 \\
\text { TS per } 10-y r \\
\text { investment period } \\
\text { ( } 576 \text { hrs total) }\end{array}$ & Various $R \& C$ & Yes & Inter-regional & [2], [10] \\
\hline $\begin{array}{c}\text { RREEOM } \\
\text { (UDel) }\end{array}$ & PJM region & Hourly over 4 yrs & Various $R \& C$ & Yes & No & [1] \\
\hline $\begin{array}{l}\text { US-REGEN } \\
\text { (EPRI) }\end{array}$ & 15 regions; US & $\begin{array}{l}\text { Hourly with } 86 \\
\text { TS/yr }\end{array}$ & Various $R \& C$ & No & Inter-regional & {$[4,11]$} \\
\hline $\begin{array}{l}\text { NEWS } \\
\text { (CIRES) }\end{array}$ & $\begin{array}{l}256 \text { load regions; } \\
\sim 37,000 \text { wind } \\
\text { and/or solar sites; } \\
\text { US }\end{array}$ & Hourly for 3 yrs & Various R\&C & Yes & Inter-regional & [5] \\
\hline $\begin{array}{l}\text { NEMS EMM } \\
\text { (EIA) }\end{array}$ & 22 regions, US & $\begin{array}{l}\text { Hourly with } 9 \text { TS } \\
\text { blocks/yr for } 20- \\
25 \text { yrs }\end{array}$ & Various $R \& C$ & Yes & Inter-regional & [12] \\
\hline $\begin{array}{c}\text { ReNOT } \\
\text { (Northrop } \\
\text { Grumman) }\end{array}$ & $\begin{array}{l}\text { Gridded cells } \\
(4 \mathrm{~km} \text { solar, } 12 \mathrm{~km} \\
\text { wind), US }\end{array}$ & $\begin{array}{l}15 \text { min solar, } \\
\text { hourly wind }\end{array}$ & Wind \& solar & No & No & {$[13,14]$} \\
\hline
\end{tabular}


At a high level, the differences among these models can be characterized by tradeoffs in temporal resolution and extent, spatial resolution and extent, and model complexity. Temporal resolution is the time step size (hourly, sub-hourly, etc.); temporal extent is the time horizon over which the model solves (1 week, 1 month, 1 year, etc.); spatial resolution reflects the handling of the wind/solar/other devices included in the model (e.g., solve site-by-site, or solve as an aggregated unit across all sites/devices uniformly); and spatial extent is the geographic coverage of the model (state, region, country, etc.). System complexity refers to the representation of different power system components, such as resource adequacy, reliability, intra-regional transmission, distribution system impacts, variability and uncertainty of renewables, and storage chronology. These "levers" can be adjusted to suit the research objective(s) and computational resources available. For instance, temporal and spatial resolution can be reduced in order to capture a greater system complexity. Most models in Table 1 have adjusted the temporal lever to include a representative subset of hours or "time slices" across a full year due to computational limits. Understanding the tradeoffs in model design can help both model builders and model users select the most appropriate combination of model parameters for their given application.

The objective of this empirical study is to quantify the tradeoffs in accuracy and computational requirements associated with the spatial and temporal modeling decision levers, which are particularly important for large-scale power system planning models. The POWER model Power system Optimization With diverse Energy Resources [6] - is used to examine these sensitivities. The complexity lever of POWER is fixed for all cases here, except where (1) the temporal treatment affects the storage formulation (Section 3.2.2) and (2) a reduced form of POWER is used to examine a fuller temporal extent (Section 3.2.3). The complexity state in the default POWER formulation includes the deployment and hourly operation of each dispatchable generator technology, storage technology, and transmission line within each representative day; variability of wind and solar generators based on hourly wind and solar resource potential data across thousands of sites; operational considerations for dispatchable generators in the form of ramp rate and minimum load limits; planned and forced outage rates; chronological storage treatment within each representative day across multiple storage technology options; interregional transmission network connecting 10 regions across the contiguous US; renewable portfolio standard (RPS) targets; generator outage rates; emissions tracking; and a statistical formulation for operating reserves including contingency, frequency regulation, and forecast error reserve requirements. POWER does not include fuel supply curves, intra-regional transmission lines, distribution system impacts (e.g., voltage deviations), or electricity market factors (i.e., market rules, market products, cash flows), and it does not make investment decisions with foresight of future policy or economic factors. An explicit planning reserve is also not included, though the load balance constraint serves as a proxy for ensuring resource adequacy across the model time horizon.

The impacts from three main sets of scenarios are assessed: (1) model simplifications and justification due to a reduced temporal resolution, (2) spatial extent and temporal size (as reflected by various representative day subset sizes) cost tradeoffs, and (3) temporal and spatial resolution cost tradeoffs. For each scenario, various RPS target levels are evaluated, ranging from $40 \%-100 \%$, and three spatial areas are considered: AllCA (California), Western 
Electricity Coordinating Council (WECC, comprised of AllCA, SW, and NW regions in the US), and the contiguous US. The results presented here focus on the higher RPS levels since actual power systems, and consequently the capacity expansion models that analyze them, are moving toward higher penetrations of renewable resources.

While computational power and data quality is improving, enabling planning models to incorporate greater detail, there remains a general lack of understanding of the sensitivity and consequences of various model adjustments. The only similar work of its kind to date to our knowledge is Mai et al. [15] and Barrows [16], which provide a systematic comparison of the impact of various capacity expansion modeling configurations and details on investment decisions and run time using the RPM model for the Western US. Other studies have examined a single aspect of the temporal treatment of capacity expansion models, such as in [17] with a new day selection method using a model of the European power system. This paper contributes to the same research area by mapping out a more extensive tradeoff space between temporal and spatial capacity expansion model components using the POWER model of the contiguous US, with additional foci on storage attributes and highly renewable electricity futures.

The purpose of this paper is not to declare technology winners and losers. Rather, the quantitative and qualitative trends from these results can help power system modelers determine the most appropriate treatment of temporal and spatial components for their application, as well as gain a better understanding of the corresponding tradeoffs in accuracy and computational requirements. This study's systematic comparison of temporal and spatial modeling tradeoffs provides a valuable contribution to the limited existing work in the literature as applied to capacity expansion modeling. However, this work does not capture the current research trend to include all energy sectors and/or novel technologies for greater system efficiency, flexibility, and synergies. Therefore, the results are likely only applicable to the narrow field of capacity expansion modeling for the electricity sector under the model assumptions embedded within the POWER model.

\section{Brief model description}

POWER consists of models of generator technologies (baseload, dispatchable, and variable), storage technologies, and a transmission network, with a statistical characterization of operating reserves. The model encompasses the ten Federal Energy Regulatory (FERC) regions in the contiguous US [18] (note that the FERC region CAISO is replaced with a modified fullCalifornia region called "AllCA"). POWER uses a full year of hourly wind and solar data across thousands of sites from 2006, historical hourly load data from 2006 by FERC region, other regional renewable resource availability data, regional cost parameters and various system parameter inputs to solve deterministically for the least-cost portfolio of generators, storage, and transmission that meet the electric load in each hour of the year while attaining a given RPS target. POWER uses these single year data inputs to construct a future electricity system with no definite time stamp and with no constraints imposed by the existing infrastructure (i.e., "greenfield" approach) except for the inclusion of existing transmission corridors. 
Each system component has an annual cost that is a function of the amortized capital costs that depend on the installed capacity decision variables, and the annual variable costs that depend on the total annual generation or storage throughput. A key model output is the total annual system cost, which is the total amortized costs of all generators, storage technologies, and transmission capacity and accounts for both fixed and variable costs, aggregated across the respective spatial extent. Total annual cost is the metric of choice for this study since it combines both the capacity and energy aspects of the system.

POWER decides how much capacity to build of each system component and how those components should be dispatched each hour. Wind and solar sites are evaluated individually, but all other components are aggregated by FERC region. POWER includes a simplified representation of three storage technologies: pumped hydroelectric storage, concentrating solar power with thermal energy storage (CSP TES), and a generic battery storage option that is indicative of the current and future range of stationary battery storage technologies. However, as noted in [6], CSP is not deployed in most scenarios evaluated with POWER due to its less favorable economics. Each storage technology is characterized by energy conversion efficiencies into and out of storage and the losses during storage. Unless otherwise noted, the results in this study are based on 8 hours of storage duration. The relevant storage costs in the total annual system costs include the power and/or energy capacity costs, which are applied to the size of the storage system built, and the variable operations and maintenance cost (VO\&M), which is applied to the energy throughput.

Additionally, there are optional submodels to include existing generators, generator retirements based on age and environmental regulations, and additional firm and flexible load from plug-in electric vehicle (PEV) charging. However, expect for a set of sensitivity runs in Appendix A, the results presented in this study do not consider the impact of PEV charging; see [6] for an investigation of the flexibility impacts of this feature. Responsive demand, new load from additional electrified sources (e.g., industrial processes and all other energy sectors), and applications for utilizing otherwise curtailable energy (e.g., district heating, irrigation pumps, water desalination/purification, and other forms of storage, such as hot and chilled water, ice, rocks, phase-change materials, and chemicals, including hydrogen) are not modeled. Emissions from fossil fuel generators are also quantified. See Chapter 4 in [7] for the full model formulation and input data details. The results presented in this study were run on a shared server with 19 nodes, 24 cores per node and 96 GB RAM per node.

A growing body of research is incorporating the above all-sector energy impacts and curtailment-reducing options into highly renewable energy system modeling analyses. For example, Jacobson et al. [19] used the LOADMATCH grid integration model to solve for a $100 \%$ wind, water, and solar resource-powered contiguous US grid to supply energy to all enduse sectors, including electricity, transportation, heating/cooling, and industry. LOADMATCH is a trial-and-error model that marches forward in time, which differs from the optimization-based POWER model that that considers many time steps simultaneously. The study also considered 
flexibility options from various storage options that utilize a variety of thermal, materials, and mechanical processes, as well as flexible electrified loads from transportation, industrial and chemical processes. Connolly et al. [20] implement a similar full-system approach, but using a step-wise process to convert to a fully renewable European system by 2050. Each step involves a fuel switch, load electrification, or other energy-saving transition. The holistic approach of connecting the electricity, heating, cooling, and transport sectors together enables greater system flexibility and efficiency. Future work should incorporate these aspects into the POWER model and tradeoff analyses of modeling methods.

\section{Tradeoffs and sensitivities of temporal modeling simplifications}

Ideally, large-scale capacity expansion models would be run serially for all hours in a year to capture the time-linked behavior of storage and to avoid omission of any solution-constraining hour(s). However, such temporal treatment is often computationally intractable. Three types of methods were explored to reduce the temporal size and/or resolution of POWER: (1) warmstarting with independent time periods and model predictive control, (2) implementing an alternating direction method of multipliers (ADMM) algorithm, and (3) running the full model with a reduced number of hours. Each of these options resulted in a loss of accuracy, in some cases at unacceptable levels. The first two methods were ruled out due to computational intractability (warmstarting) or accuracy losses (ADMM). Details on these methods and results are provided in Section 4.10 of [7].

In the third method - running the model for a reduced number of hours - two cases were evaluated: (1) using a coarser temporal resolution of all days within a year, and (2) using a representative subset of days from a full year. Similar, but varying, methods have been used in most other large-scale capacity expansion models to select a representative subset of hours or "time-slices." These include peak and median load-based days from within each month (SWITCH), aggregate time-slices across each season plus a summer-peak time-slice (ReEDS), and extreme events within "bubbles" at each of the eight Euclidean corners of the joint loadwind-solar distributions with additional hours to represent base and shoulder events (USREGEN).

\subsection{Coarser temporal resolution}

The first set of reduced-hours model modifications included all days of the year with reduced temporal resolutions in POWER. The resolutions considered were every 2 hours, 4 hours, and 8 hours across the full year. The time series data for these runs were created by averaging all hourly values within each time step block. POWER maintained full chronological treatment in these runs, with uniform time step weights equal to the time step size (e.g., weight of 2 for 2 hourly resolution). Results from these model instances are presented in the temporal resolution results in Section 4.2. 


\subsection{Representative subset of days}

The second reduced-hours modification used a representative subset of days within a full-year temporal extent. This modification results in the loss of chronology across all resulting hours. Instead, chronology is maintained only among the hours within each representative day. POWER still looks across the full temporal extent of one year for capacity decisions, however.

The sensitivity of the model to the day selection method and associated storage model modifications are investigated in Sections 3.2.1 and 3.2.2, respectively. The impacts of these modeling modifications are then quantified and justified with a reduced form model of POWER in Section 3.2.3. The full version of POWER with a representative subset of days was used for all results in Section 4, except the temporal resolution results in Section 4.2.2.

\subsubsection{Day selection method}

The representative subset of days for POWER include "extreme" event days (high and low), and if desired, additional days selected randomly to represent "typical" system behavior. Extreme days contain the maximum hour along each of the eight vectors from the origin to the vertices of a unit cube, based on normalized hourly wind and solar (averaged across all potential developable sites) and load data for a full year for each region and interconnected aggregate area. Extreme days are first selected, and then random "typical" days are added until the desired number of total days is reached. In this way, the most constraining instances are first included so that a more (or equally) optimal solution is obtained as random days are added. In order to calculate annual total values, weights were then assigned to each selected day using least-squares to best match the full-year wind, solar, and load distributions. One important caveat is that these weights are based on the maximum hourly production across all wind and solar sites and only the historic hourly load; they are not updated based on any new wind or solar builds or modified load shapes. Additional steps were implemented for scenarios with interconnected regions to further reduce the representative subset of days into a more concise subset; various methods were tested to determine the best option for different spatial extents. More details on this methodology and corresponding sensitivity results are provided in Sections S1.1 and S1.2 of Appendix A.

The main finding from the comparison of different day selection methods for interconnected regions is that the model outputs are sensitive - for some spatial extents and cases more than others - to the subset of days included in the temporal extent. For example, results from the interconnected WECC region yielded $<15 \%$ difference in total cost, but up to double the renewable overgeneration values, between different day selection methods and subset sizes (see Section S1.2). The key is to capture the extreme events days without over-representing their impact on the system performance (e.g., renewable overgeneration and energy production). Similar observations of this sensitivity to the day selection methodology were noted by the USREGEN model [21] and in a paper presenting a methodology for selecting representative days based on a modified hierarchical clustering technique [17]. Possible methods of addressing this issue include clustering algorithms such as k-means or hierarchical clustering, which have shown 
success in finding representative subsets of electric load data (e.g., [22] using load and wind data with validation against full-year data, and [23] using an order-specific clustering algorithm to accommodate sequential load data, while preserving its daily fluctuations) and jointly for electricity prices and solar irradiation data [24].

\subsubsection{Sensitivity to storage model modifications}

The baseline version of POWER uses a representative subset of days that are distributed throughout a full year. Because of this temporal disjointedness, the chronological tracking of storage is limited to the 24 hours within each representative day. To deal with the seams between the days, a periodic boundary constraint is applied to each representative day so that POWER chronologically tracks the amount of storage going into, out of, and held in storage for each time step within each day. Each storage technology is tracked separately and represents the aggregate capacity of that technology within each spatial region (i.e., individual battery sites are not modeled). The model results are sensitive to the way in which this periodic boundary constraint is applied. Existing methods to handle this discontinuity focus on balancing the storage energy over each day, either by requiring that (1) the sum of the net storage values equal zero each day (e.g., [2]), or (2) the amount of energy held within storage is the same at the start and end of the day (e.g., [25]). These methods are described more fully in Section S1.3 in Appendix A.

A comparison of the performance of these two methods under different storage balancing conditions was made using the subset of days from Section 3.2.1 for highly renewable AllCA and WECC systems. These storage balancing conditions included summing the net storage values to zero each day (method 1), as well as different capacity levels to start/end each day (method 2). The results between all of these conditions generally yielded $<3 \%$ difference in total annual system cost. A comparison of these conditions for AllCA with PEV load and 56 representative days at an 80\% RPS level is shown in Figure S5 in Appendix A; the storage component of this system consists of about $12 \mathrm{GW}$ power capacity and $93 \mathrm{GW}$-hr energy capacity of pumped hydro storage, corresponding to about $8 \%$ of the total system installed capacity. However, the best option on the combined bases of cost, renewable overgeneration, run time, and daily storage profile is to require that the system holds the same amount of energy within storage at the end of each day (method 2) at a level chosen endogenously by the model. See Section $\mathrm{S} 1.3$ in Appendix A for more details on these results. This storage model modification was implemented in all final model runs that used a representative subset of days in Section 4.

To assess the sensitivity of POWER to the daily storage balance constraint, the chosen daily storage balance constraint was then compared with the original full chronological treatment for a springtime period of 77 days. As shown in Figures S6-S7 in Appendix A, constraining storage to balance each day yielded very similar system portfolio and total system costs $(<2 \%$ difference $)$ as the full chronological version. However, the daily storage balance constraint case yielded noticeably more renewable overgeneration, more ramping of generators, and greater throughput in storage than allowing inter-daily storage flows (chronological version), especially in a fully renewable AllCA system. See Section S1.4 in Appendix A for more details on these results. 
The sensitivity of the model to the presence and/or choice of this daily balance constraint further highlights the significance of proper temporal handling in power system planning and grid integration models, especially at high RPS targets when storage tends to play a greater role. Awareness and proper treatment of these modeling features will therefore become more important as capacity expansion models investigate higher penetrations of renewable resources. Ideally, a full-year serial model would be used to capture a more accurate performance, and likely a greater benefit, from the seasonal and/or weekly flexibility that storage can provide (e.g., a weekly storage cycle is observed in many pumped hydro storage facilities). However, based on the results here, the small difference in system buildout and costs justifies the use of a daily storage balance constraint when the daily seams are properly handled and when computational limitations prevent the implementation of a full-year serial treatment.

These storage sensitivity results reflect outputs with a simple storage model for a limited suite of storage technologies. While POWER does account for the diurnal and seasonal variations in solar resource supply for CSP TES, the results here do not reflect additional sensitivities to the storage duration (assumed to be 8 hours), diversity of battery storage options, or the impact that additional storage technologies, new load, or responsive demand might have. The results here are intended to highlight the importance of the way in which a storage balance constraint is applied with a simple storage model in a highly renewable system when full chronological treatment is computational intractable.

\subsubsection{Sensitivity to the number of representative days}

To examine the sensitivity of model accuracy and computational requirements to the number of representative days implemented, a reduced form model was created for the AllCA region. This simplified version of POWER contained only natural gas, wind (onshore and offshore with a uniform buildout across all sites), solar PV (large-scale and residential with a uniform buildout across all sites), battery storage, reserve requirements, and emission components from the original full model formulation. These simplifications enabled an ideal full-year serial run as a benchmark for comparing the same full-year temporal extent with various representative day subset sizes (8 to 168 days) with the daily storage balance constraint that was introduced in Section 3.2.2.

For each representative day subset size that included both extreme and random days, ten instances were run and the results then averaged. The extreme-only 8-day scenario and full-year serial 365-day scenario each had only a single set of results. Figure 1 displays the resulting total annual system cost, standard deviation of cost across the 10 instances, and renewable overgeneration for various representative day subset sizes for AllCA with an 80\% RSP target. Figure 2 plots the percent loss in objective function accuracy (total system cost) relative to the 365-serial-day target, as well as the computational load measured as CPU run time, for these same representative day subset sizes. For comparison, the maximum memory usage (not shown) also scales roughly quadratically with the number of days, requiring about 55GB of RAM in the 365-serial-day instance.

The total cost, standard deviation of cost, accuracy loss, and renewable overgeneration all decrease with increasing representative day subset size, each approaching the target 365-serial- 
day result. This trend is only approximate, due to the variation in randomly selected days, especially at lower subset sizes where the standard deviation is higher. Nevertheless, these results reveal the diminishing returns in accuracy (roughly logarithmic) and increasing computational run time (quadratic) with increasing subset size. For comparison, the maximum memory usage also scales roughly quadratically. The loss in objective function accuracy was generally $\leq 6 \%$ for the representative day subsets of all extreme plus some random days (i.e., 14+ days).

The quadratic run time observations from this reduced form version of POWER is supported by well-documented findings from early work with the simplex method, which show that linear programs can generally be solved in "polynomial-time" [26] or in some cases "exponential-time" [27]. See Section S15 in Appendix A for a simple qualitative example that helps to describe these computational run time trends.

Few papers have evaluated the tradeoffs in accuracy and/or run time with various modeling structures as applied to power system planning. Among them, Nahmmacher et al. in [17] similarly compared cost versus the number of representative days using the LIMES-EU model of the European power system. Their total system cost results, however, increased with increasing subset size, whereas those with POWER decreased. The reason is discussed shortly. Similarly, Nicolosi [28] compared overgeneration by the number of time slices using an investment and dispatch model of ERCOT and found that overgeneration increased with a larger number of time slices; the opposite trend was observed with POWER in Figure 1. The difference between the results from Nahmmacher et al. and Nicolosi and those here in Figure 1 can be attributed to the absence or limited inclusion of extreme days in their day selection methodology. Conversely, the day selection method in POWER begins with extreme days and incorporates more random "typical" days with increasing subset size, consequently yielding more cost-effective systems and lower overgeneration. This observation follows linear programming theory, by which a subset of the full problem (solution space) will yield a suboptimal (or equal to the optimal) solution.

Similar accuracy and run time results as those in Figure 2 were shown in Mai et al. [15], who found that increasing the number of hours included in the dispatch period from 24 hours to 96 hours increased computational run time by nearly 2 orders of magnitude (from about 1.5 hours to 7 days, respectively), with little impact on the investment decisions using the RPM model of the US Western Interconnection. Follow-on work by Barrows et al. [16] with the RPM model found that using average daily profiles, where each hour in a 24-hour period represents the average across those same hours in a full week, represented variable generation more accurately but yielded marginally larger capacity expansion errors than with the same number of non-averaged 24-hour sample periods. Other studies similarly commented on the impact of the way in which fluctuations in load and variable generators are captured. A smaller number of time steps underestimates the variability and occurrence of extreme load/wind/solar events, which overestimates the value of baseload units and underestimates the value of flexible resources such as natural gas peaking plants and storage, as shown by Kannan and Turton [29] using the TIMES electricity system model applied to Switzerland and Nicolosi [28] for ERCOT. The differences between the results in these studies highlight the importance of selecting appropriate representative days, which varies by model and application. 
There are two key takeaways from the results using the reduced form model of a single region (AllCA) without transmission, along with the findings of the day selection and storage balance methods. First, while sensitive to the exact way in which these components are handled, using a representative subset of days with an associated daily storage balance provides a reasonable suboptimal approximation in this linear programming power system planning model. This suggests that comparable time step reduction assumptions may be also justified for similar models. Secondly, while likely sensitive to the day selection method and level of model complexity, the qualitative trends observed in Figure 2 can help inform the choice of the ideal day selection size for similar linear programming modeling exercises.

The exact error cannot be quantified for the full serial year version of POWER, but accuracy losses caused by solving across a smaller set of representative days with the reduced form model are small, generally $\leq 6 \%$. Thus, when accuracy requirements are comparably low, the small accuracy losses from using a representative subset of days are justified by the computational savings. Any residual error from these representative day method and subset size choices are likely outweighed by other general sources of model errors (e.g., input cost data, system parameters, etc.). However, accuracy results will vary for different models based on their assumptions for temporal, spatial, and complexity factors. This $6 \%$ value should not be interpreted as a universal standard or result.

The ideal day selection method and size will vary by model, though similar qualitative trends in accuracy tradeoff should be found for other linear models employing a comparable hybrid extreme + random day method, given the fundamental principles of linear programming. Future work should further investigate the accuracy result impacts from various modeling assumptions, such as the storage model formulation, cost assumptions, variable renewable curtailment treatment, and inclusion of multiple energy sectors. As previously mentioned, additional work is also needed to develop more robust day selection methods, particularly with the capability to capture the extreme events without over-representing their impact on the system performance. Ideally, this future effort will also develop methods or metrics for determining the ideal size of the representative day set, given the properties of the study area and the model configuration. 


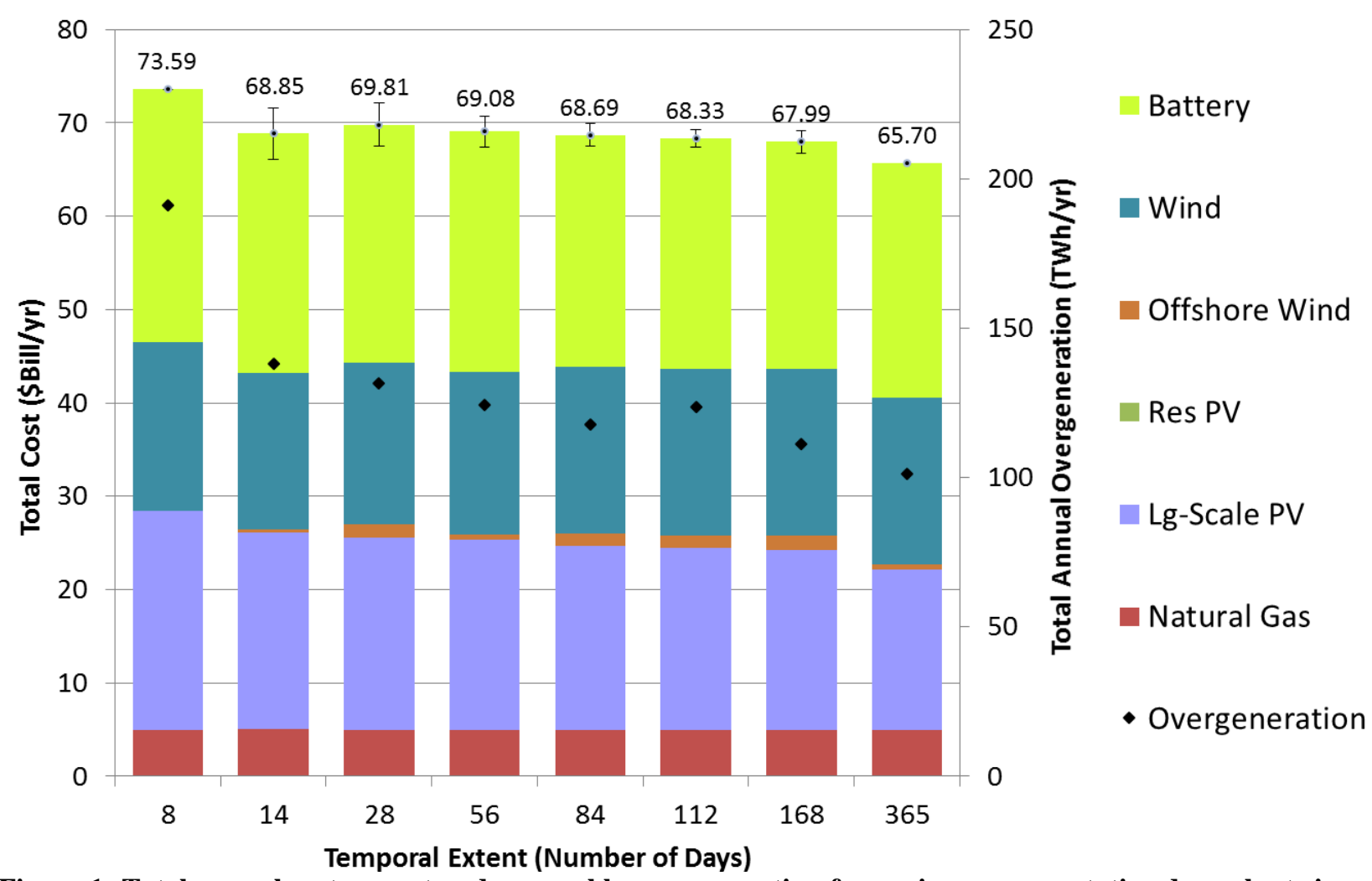

Figure 1 Total annual system cost and renewable overgeneration for various representative day subset sizes for the AllCA region with a reduced form model at an $80 \%$ RPS target. Values for 8 days are based only on the most extreme wind, solar, and load events. Values for all other days (except 365-serial-days) are based on the average of 10 runs each, which include the same 8 extreme days plus additional random days. Total costs (2006USD) are shown above bars, and error bars correspond to standard deviation of total costs.

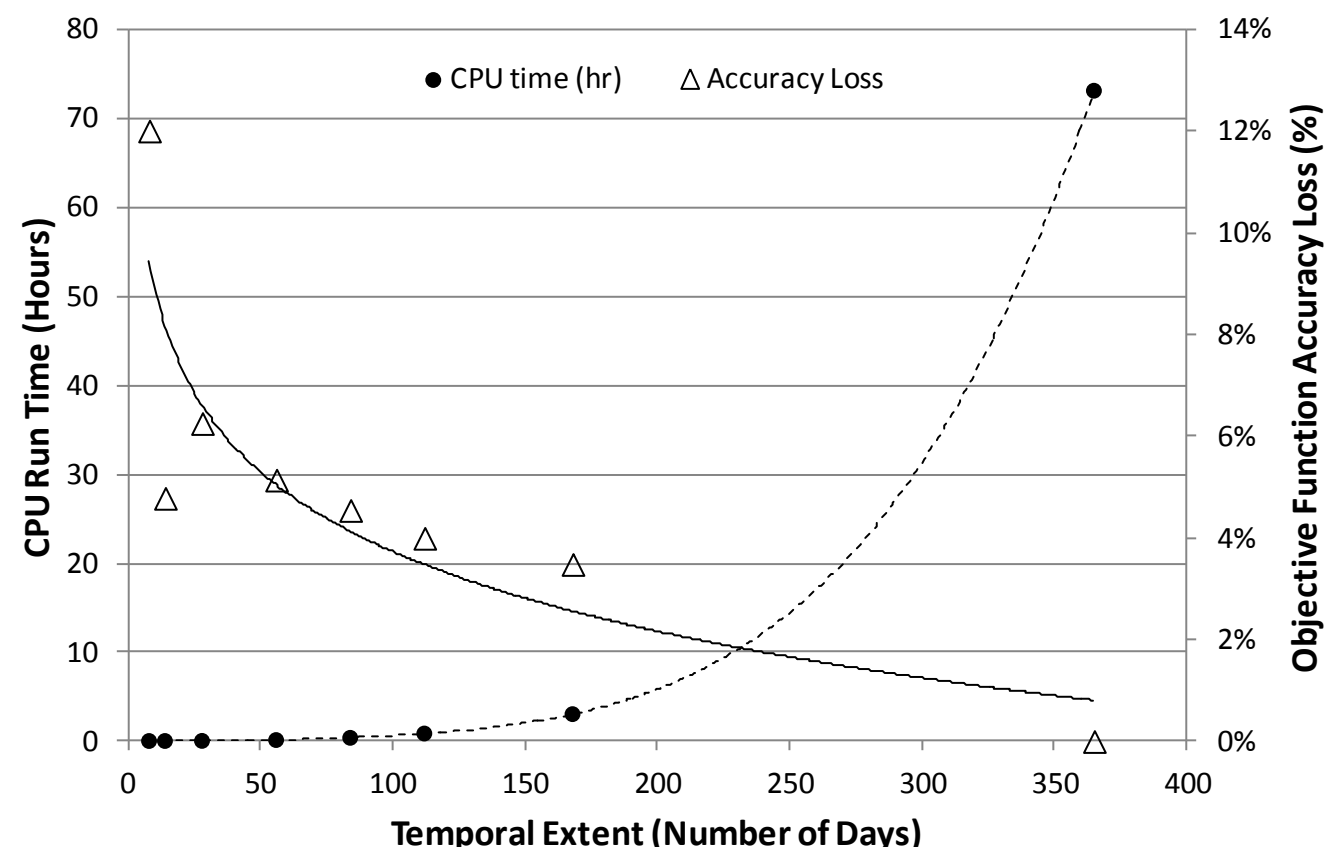

Figure 2 Computational load and accuracy loss for various representative day subset sizes for the AllCA region with a reduced form model at an $\mathbf{8 0} \%$ RPS target. Run time scales quadratically with increasing subset 
size, and accuracy (relative to 365-serial-day target) increases roughly logarithmically with increasing subset size. The number of decision variables and constraints strongly depend on the subset size.

\section{Tradeoffs between modeling decision levers}

Various scenarios were completed for the AllCA FERC region, WECC, and the contiguous US to evaluate the tradeoffs in temporal and spatial modeling decision levers. All model results presented here assume 5\% intra-regional transmission line losses and 8 hours of storage. Existing and retiring generators are not considered.

\subsection{Representative day subset size and spatial extent}

The temporal size and spatial extent tradeoffs were evaluated using a representative subset of 8 days ( 1 week), 28 days ( $\sim 1$ month), 56 days ( $\sim 2$ months), and 168 days ( $\sim 6$ months) for the AllCA (2006 peak load $\sim 62 \mathrm{GW}$ ) and WECC (2006 peak load $\sim 133 \mathrm{GW}$ ) spatial extents. See Sections 3.2.1 and S1.1 for details on the selection of these days. Ten instances were run for each representative day subset size beyond 8 days for AllCA and 5 instances for each extent beyond 22 days for WECC. All results presented here are the averages of those instances. Additional spatial extent results are presented for the full contiguous US (2006 peak load $\sim 729 \mathrm{GW}$ ). All runs in this section include the daily storage balance constraint (Section 3.2).

Figure 3 summarizes the total annual system cost for each temporal and spatial extent combination for AllCA and WECC. Also shown are the percent increases in cost relative to the most "ideal" computationally tractable combination in each spatial extent column (open star). The most ideal representative day subset size for each column is a full year (filled star), but this was computationally intractable. The spatial extent columns (from left to right) represent the AllCA region individually, the sum of independent WECC constituent regions (AllCA, NW, and $\mathrm{SW}$ ), and the interconnected WECC region. Each increasing spatial extent required greater computational resources, which reduced the maximum achievable representative day subset size.

On the spatial extent axis, the total cost was sensitive to the impact of geographic aggregation. This is reflected by the difference between the sum of WECC regions and the interconnected WECC, with roughly a $14 \%$ reduction in total cost due to interconnection for the 56 representative day subset size. Additional runs for the contiguous US (Section 4.1.2) reveal that the benefits of aggregation are increased for larger spatial extents and RSP targets, achieving total cost reductions of about $42 \%$. The reliability and cost benefits of aggregation are welldocumented for cases where geographic areas are interconnected, allowing for access to a greater diversity of resources and reserves (e.g., [30] with an extension of the Western US Energy Imbalance Market and [31] with a US-based wind study). This study reveals that the cost trends with increasing spatial extent are driven by these aggregation benefits rather than by an underlying computational tradeoff. 


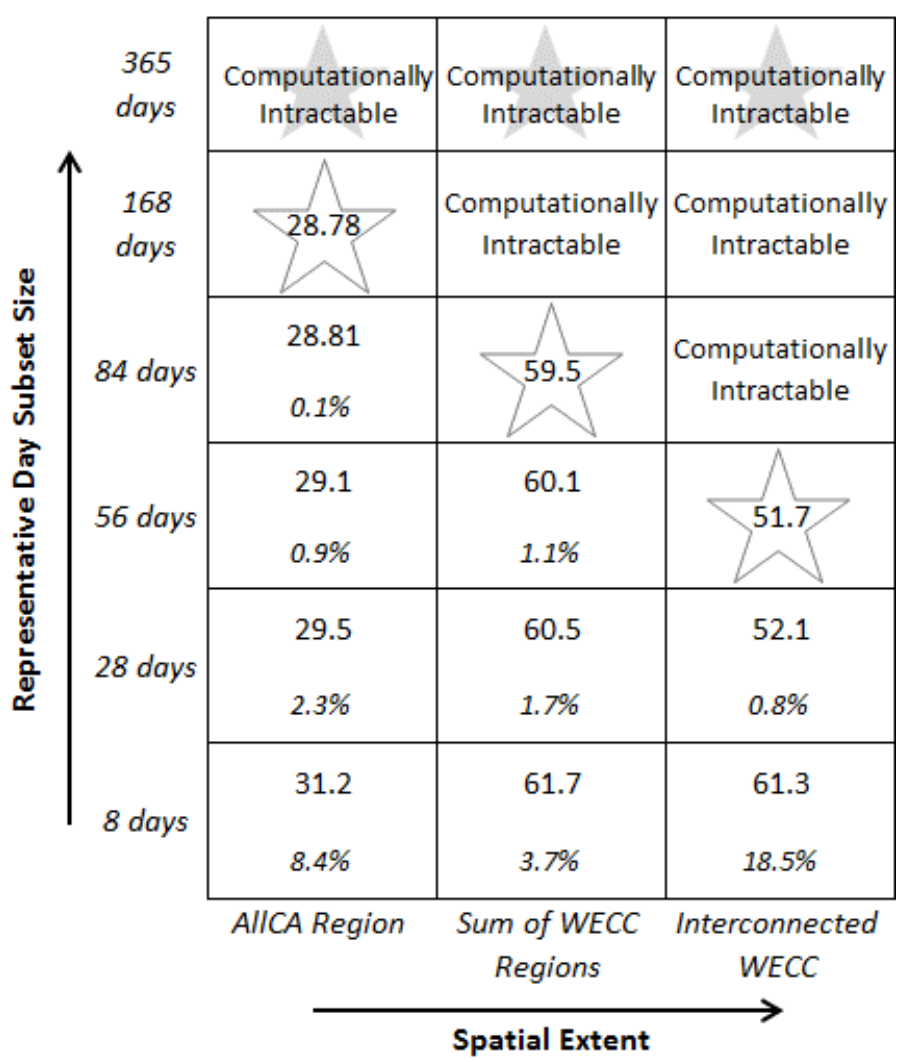

Figure 3 Temporal and spatial extent cost tradeoffs for AllCA and WECC regions at an 80\% RPS target. Total annual system cost (Billions of 2006USD/year), with percent increase in cost in italics relative to the most "ideal," computationally tractable case within each spatial extent column (open star). The ultimate goal is to model each spatial extent category for a full year of days (filled star), but this was computationally intractable.

\subsubsection{Representative day subset size}

As shown in Figure 3, the total cost decreased as the representative day subset size increased, since the smaller subset sizes contain disproportionally more high-load hours (see Sections 3.2.1 and S1.1). Increasing the size of the subset by adding more random "typical" days resulted in an increasingly more accurate representation of the full year.

The representative day subset sizes in Figure 3 followed the same high-level trends as the reduced form model in Section 3.2.3 for total cost, standard deviation of total cost, renewable overgeneration, and tradeoffs in computational requirements and accuracy (see Figures S9-S13 in Appendix A). In the AllCA system, 56 days provided the best combination of accuracy, computational requirements, and system configuration and operation. Because the standard deviation of total cost was very small, any single instance from among the ten 56-day runs would suffice, but the most representative instance was used for all subsequent AllCA scenarios.

A similar representative day subset size threshold was determined to be suitable for a large-scale linear optimization model of the European electricity system [32]. They found that computations limited to 6 typical weeks (42 days) were sufficient; a sensitivity analysis with larger subset sizes did not significantly change results. 
For the larger spatial extent of WECC, 28 days was determined to be the ideal representative day subset size. Similar observations were made for these WECC cases as with the single AllCA region. See Section S2.2 in Appendix A for details on these AllCA and WECC results.

\subsubsection{Spatial Extent}

The sensitivity to spatial extent was examined by comparing the total system cost breakdown and renewable overgeneration of the independent constituent WECC regions against the interconnected WECC case at 50\% and 80\% RPS targets (Figure 4). Region-specific details of these results are shown in Figure 5 and Figure 6 for the independent regions and Figure 7 and Figure 8 for the interconnected scenario.

The cost breakdown in Figure 4 reveals that the interconnected case deployed less solar, wind, and storage than the independent region case for both RPS targets. At a higher RPS target, there was significantly more renewable overgeneration, but also greater relative cost and overgeneration savings, with aggregation. Additionally, as RPS requirements increased, wind was built out before solar, especially in the interconnected system. These findings agree with the base case results of a WECC study using the SWITCH model [2], although the SWITCH model installed small amounts of coal and nuclear, while the results here had none of either.

New transmission capacity occupied a small fraction of the interconnected system total cost. Both the 50\% and $80 \%$ RPS interconnected WECC scenarios utilized transmission, but only the $80 \%$ case required new additional capacity, representing about $1 \%$ of the total cost. This finding roughly agrees with other grid integration studies of the western US, such as that using the SWITCH model [33] and that using the WREZ model [34], both of which found that new and existing transmission costs represent less than 10-19\% of the total cost for a highly renewable system. Transmission costs presented here were reasonably smaller since they were based only on new (and not total) capacity. To calculate the regional transmission costs in POWER, the cost for each transmission segment was assumed to be split evenly between the two end node regions. 


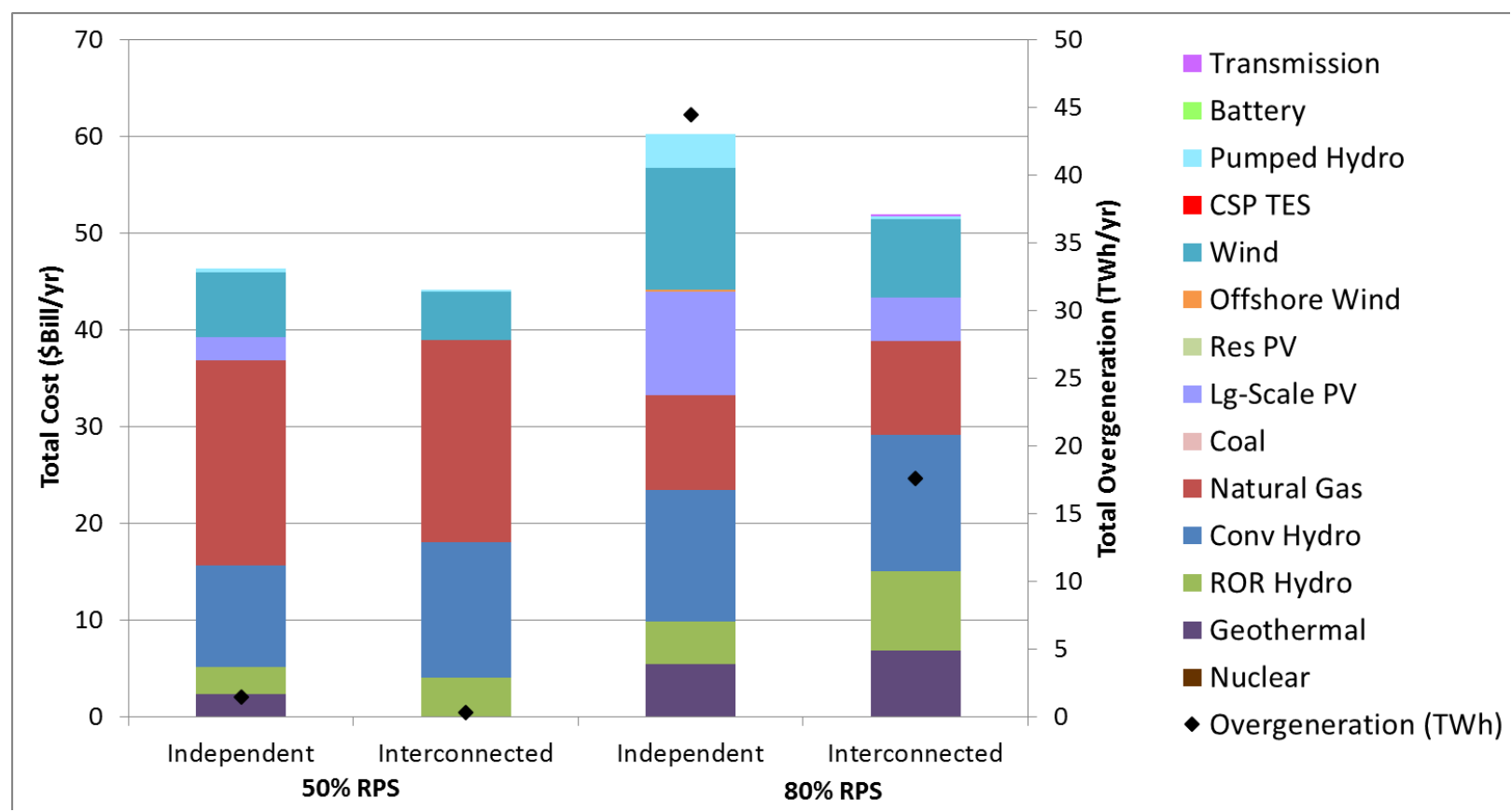

Figure 4 Total annual system cost and renewable overgeneration for independent WECC regions vs. interconnected WECC (AllCA, SW, NW) for 50\% and 80\% RPS targets. All values are for cases with a representative subset of 56 days. Costs are in 2006USD. 


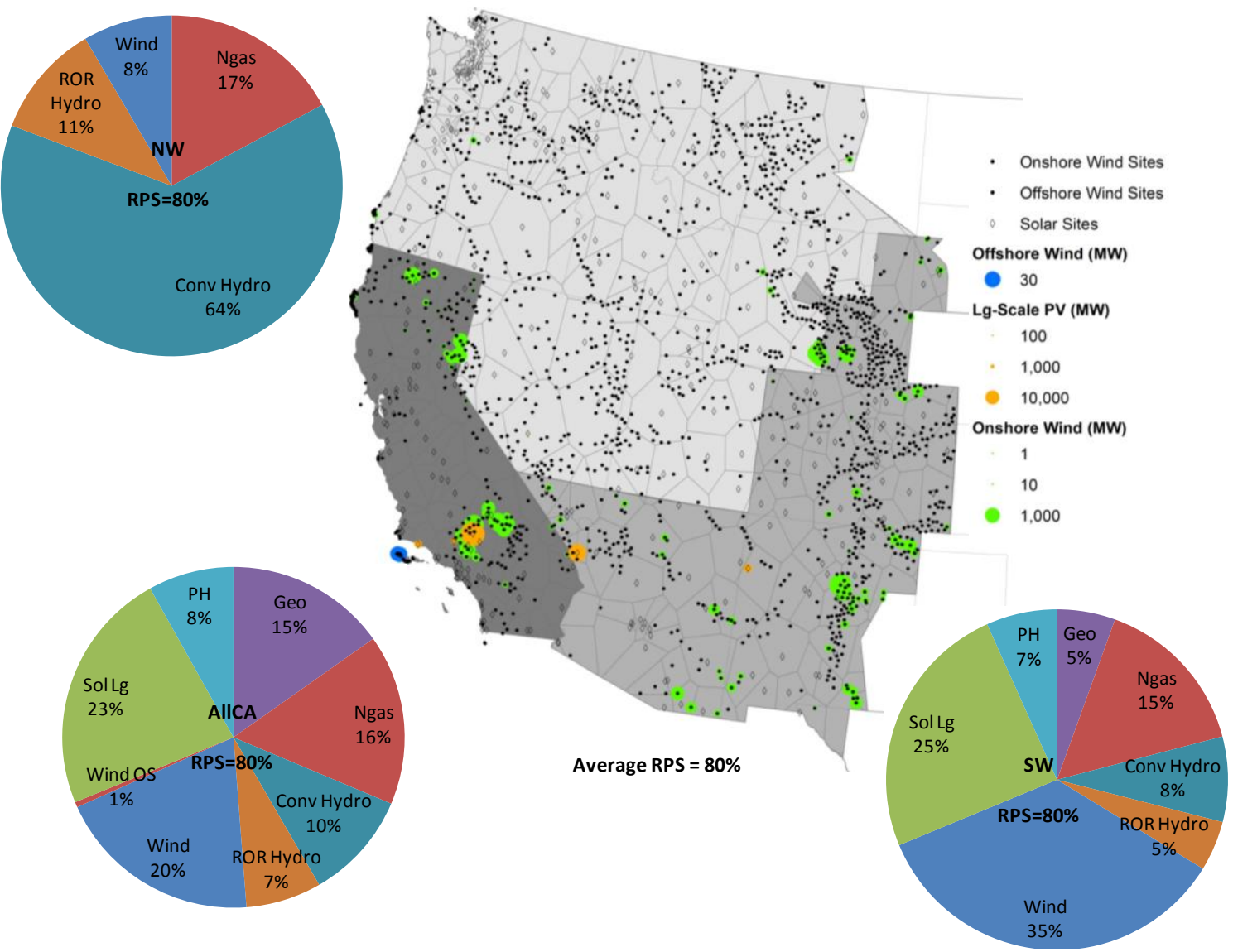

Figure 5 Breakdown of total annual system cost and wind and solar site buildouts for independent WECC regions (AllCA, SW, NW) at an $\mathbf{8 0 \%}$ regional RPS target. Pie charts show relative contribution of each built generator and storage technology to the total annual cost in each region. Actual achieved RPS in each region is shown in pie charts; average of all regions is shown at bottom. Results are based on a representative subset of 56 days.
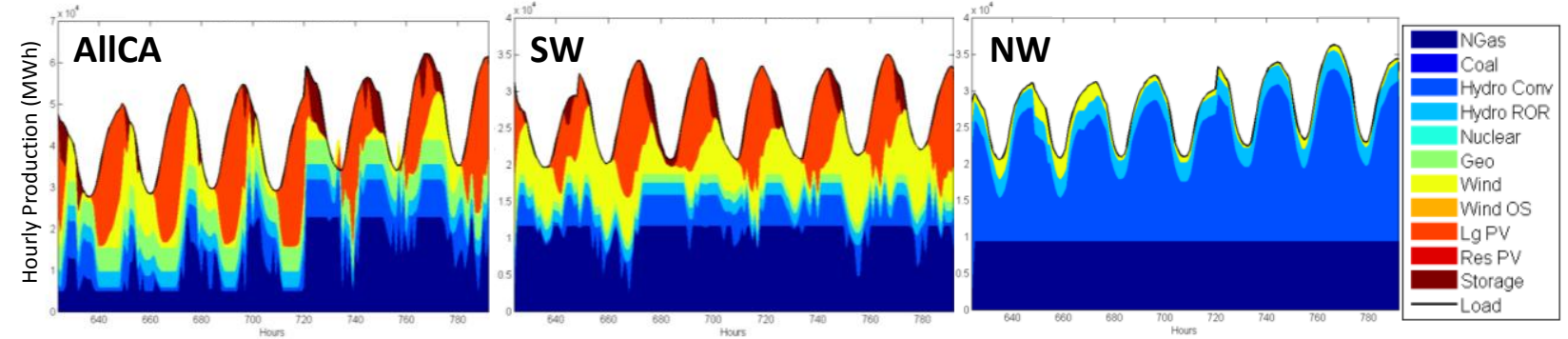

Figure 6 Zoom-in to 8 days of system performance for independent WECC regions at an 80\% RPS target. Days shown are from the months of June and July (from a representative subset of 56 days) and correspond to results shown in Figure 5. 


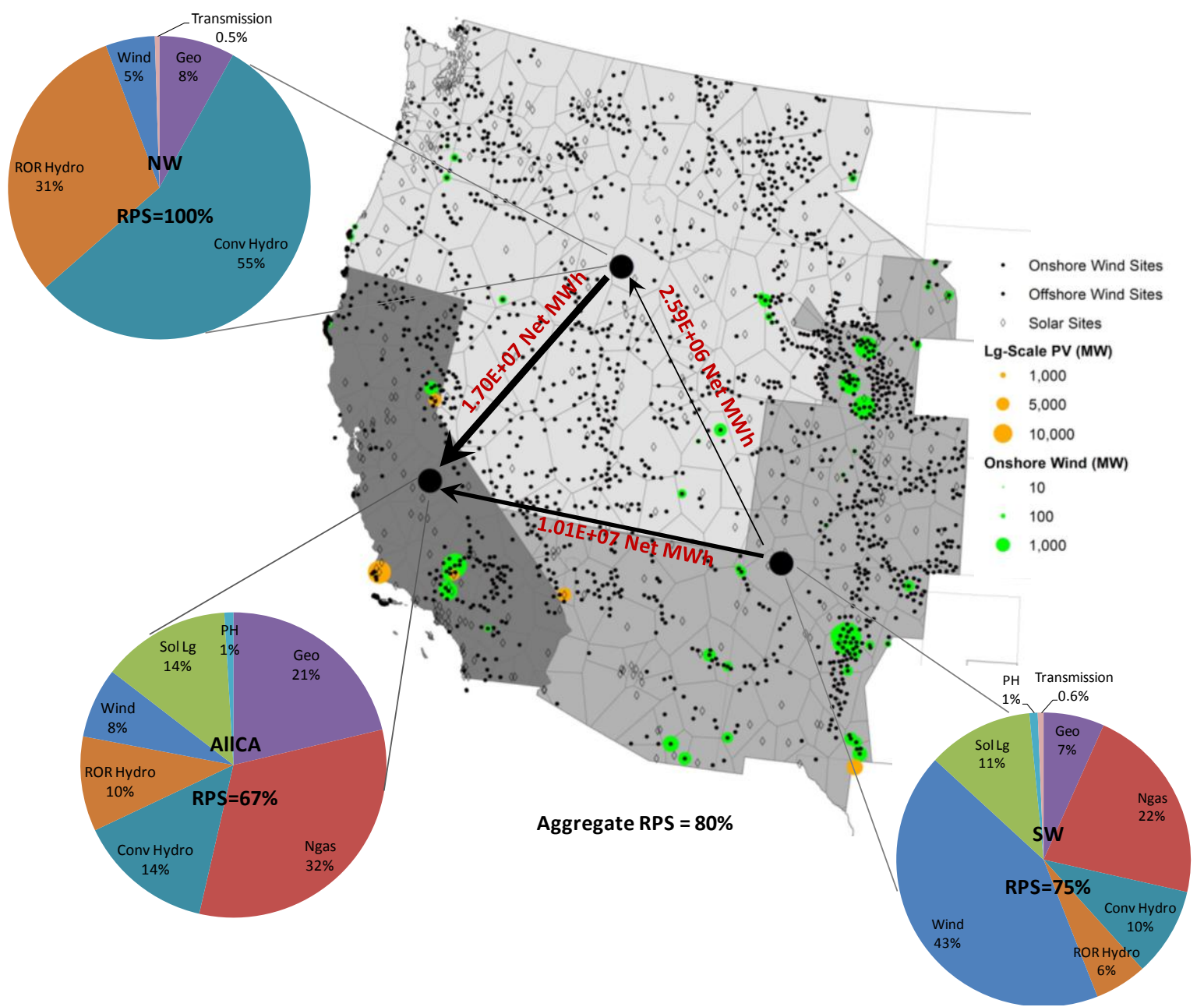

Figure 7 Breakdown of total annual system cost, wind and solar site buildouts, and net transmission flows for interconnected WECC with an 80\% aggregate RPS target. Pie charts show relative contribution of each built generator and storage technology to the total annual cost in each region. Arrow show dominant direction of transmission flow, and weight of line shows relative magnitude of net flow. Actual achieved RPS in each region is shown in pie charts; actual aggregate RPS is shown at bottom. Results are based on a representative subset of 56 days.
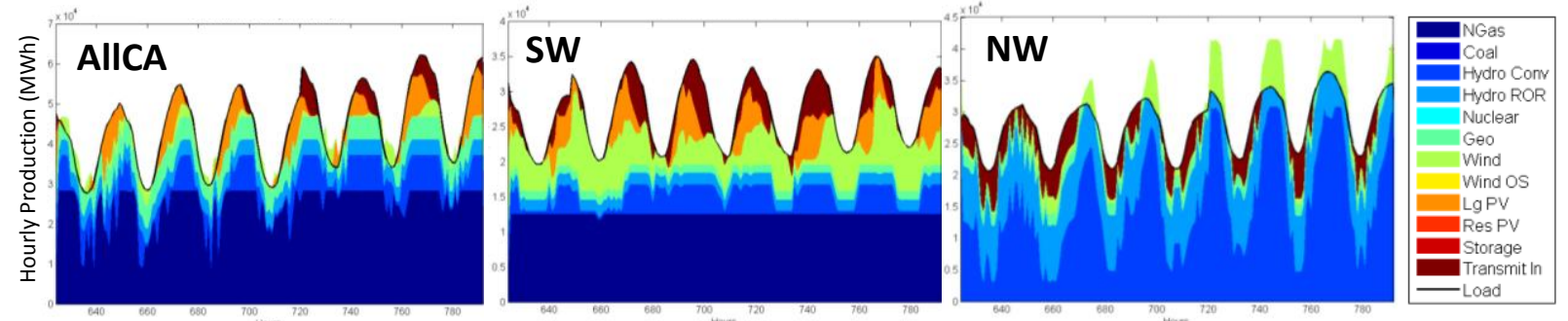

Figure 8 Zoom-in to 8 days of system performance for interconnected WECC regions at an 80\% RPS target. Days shown are from the months of June and July (from a representative subset of 56 days) and correspond to results shown in Figure 7. Note the difference in color bars between Figure 6 and this figure.

The wind and solar site buildouts, transmission flows, relative cost contributions (Figure 5 and Figure 7), and system performance (Figure 6 and Figure 8) between the independent regions and interconnected scenarios reveal several important differences in the effect of aggregation as part 
of the spatial extent spectrum. The first difference was the disproportionate contribution of the constituent regions to the aggregate RPS, suggesting the need for regional-and-resource-specific RPS targets. An $80 \%$ aggregate RPS does not necessarily mean that each region will achieve an $80 \%$ RPS. In fact, in the results of this WECC system, both AllCA and SW built and used more natural gas generators in the aggregated case, resulting in lower regional achieved RPS targets (67\% and $75 \%$, respectively) as compared to the independent region case (each at $80 \%$ ). The NW region compensated for this in the aggregated case by becoming fully renewable and transmitting excess generation (Figure 8). The dominate presence of hydro in the NW region poses a potential reliability risk in the event of reduced hydro flows due to drought or other causes.

Secondly, there was less solar deployment in the aggregated case. This was especially apparent in the performance time series plots (Figure 6 and Figure 8). All developed solar capacity was large-scale PV; no residential PV was built in any cases, including other model scenarios of the contiguous US. Large-scale PV is an abundant, comparatively lower-cost resource, precluding the need for any residential PV with this model formulation. However, this does not suggest that residential PV should be disregarded for highly renewable systems. For example, if the intraregional transmission and distribution line losses and capacity savings are considered, then residential PV may become a more cost-effective option. These benefits are outside of the spatial resolution captured by POWER, but separate sensitivity analyses of the US and AllCA systems with POWER revealed that a 40-45\% reduction in residential PV costs resulted in the displacement of roughly $10-18 \%$ of large-scale PV capacity and about $2-7 \%$ of wind capacity by residential PV, relative to an $80 \%$ RPS target base case without PEV load. In these sensitivity cases, the extreme short-duration events caused by the overall larger share of coincident solar production resulted in a greater need for system flexibility, fulfilled by storage and curtailment (see [6] and Section 5.4 in [7]).

Third, based on these results from the western US with POWER, aggregating regions through an enhanced transmission system is economically more favorable than only harnessing local resources coupled with storage. Storage was still used with transmission, but to a significantly lesser degree, especially at higher RPS targets. For instance, in the WECC scenarios with an $80 \%$ RPS target, energy from pumped hydro storage met about $3 \%$ of the total load in the independent regions case, but only $0.2 \%$ in the aggregated case, with transmission instead providing $6 \%$ of the total load in the latter case. Transmission occupied $<1 \%$ of the total cost in this WECC scenario. The dominant transmission flows agreed relatively well with the findings from the SWITCH model [2], where a net flow from wind generation in the SW was transmitted to the NW, net flow from hydro in the NW was transmitted to AllCA, and net generation from solar and wind in the SW was transmitted to AllCA. However, noticeably less solar was installed in the SW region with POWER.

To determine if these same aggregation effects transfer to the rest of the US, POWER was then run with a subset of 14 days for all ten FERC regions, both independently and as an interconnected entity comprising the full contiguous US, at $80 \%$ and $100 \%$ RPS targets. This reduced representative day subset size was necessary due to the significant increase in computational memory requirements with the larger US system. The resulting total cost 
breakdown by system component is shown in Figure 9 for independent FERC regions and in Figure 10 for the interconnected scenario. 


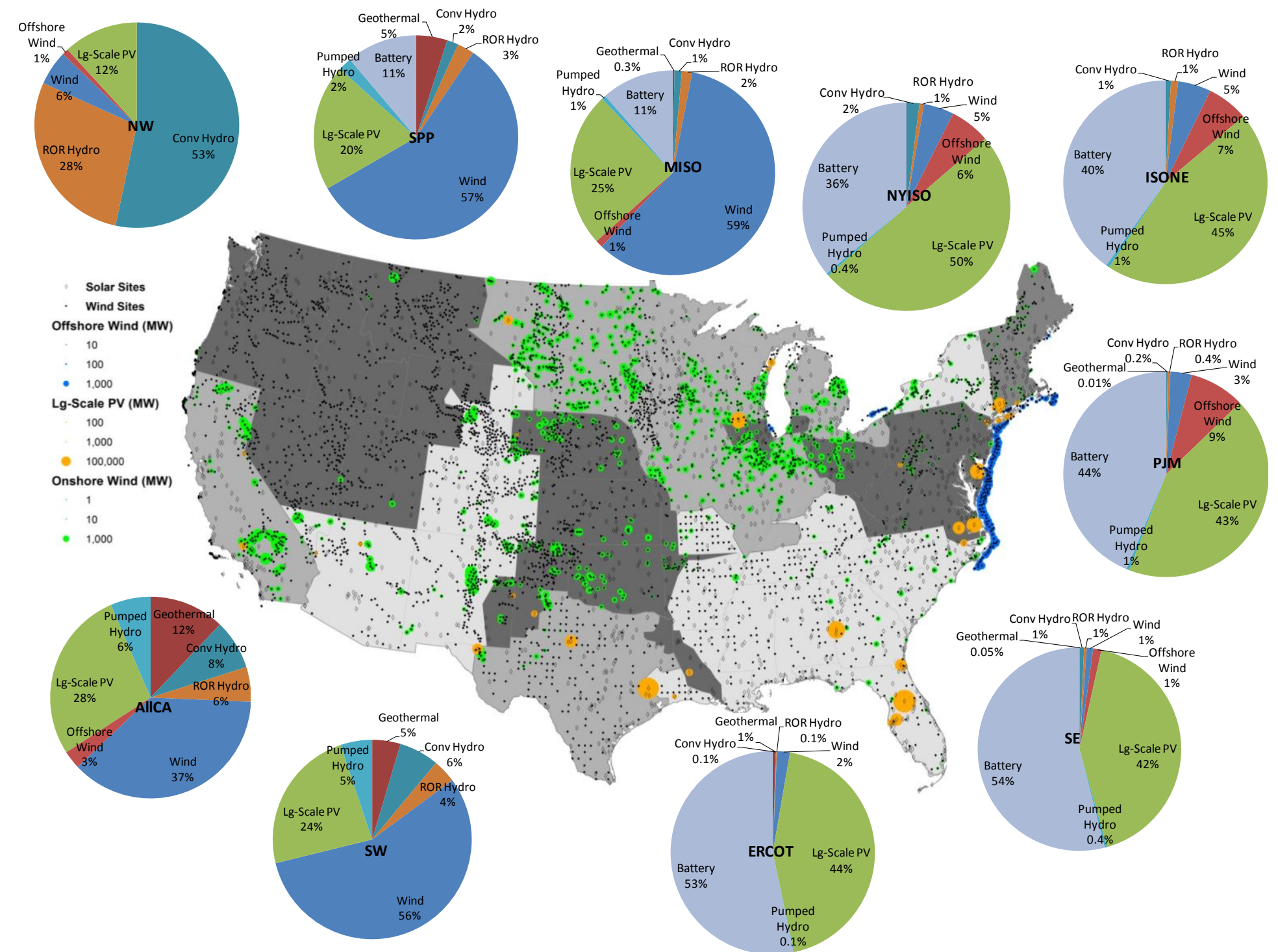

Figure 9 Breakdown of total annual system cost and wind and solar site buildouts for All FERC regions at $1000 \%$ RPS target. Pie charts show relative contribution of each built generator and storage technology to the total annual cost in each region. Actual achieved RPS in each region is $100 \%$. Results are based on a representative subset of 14 days. 


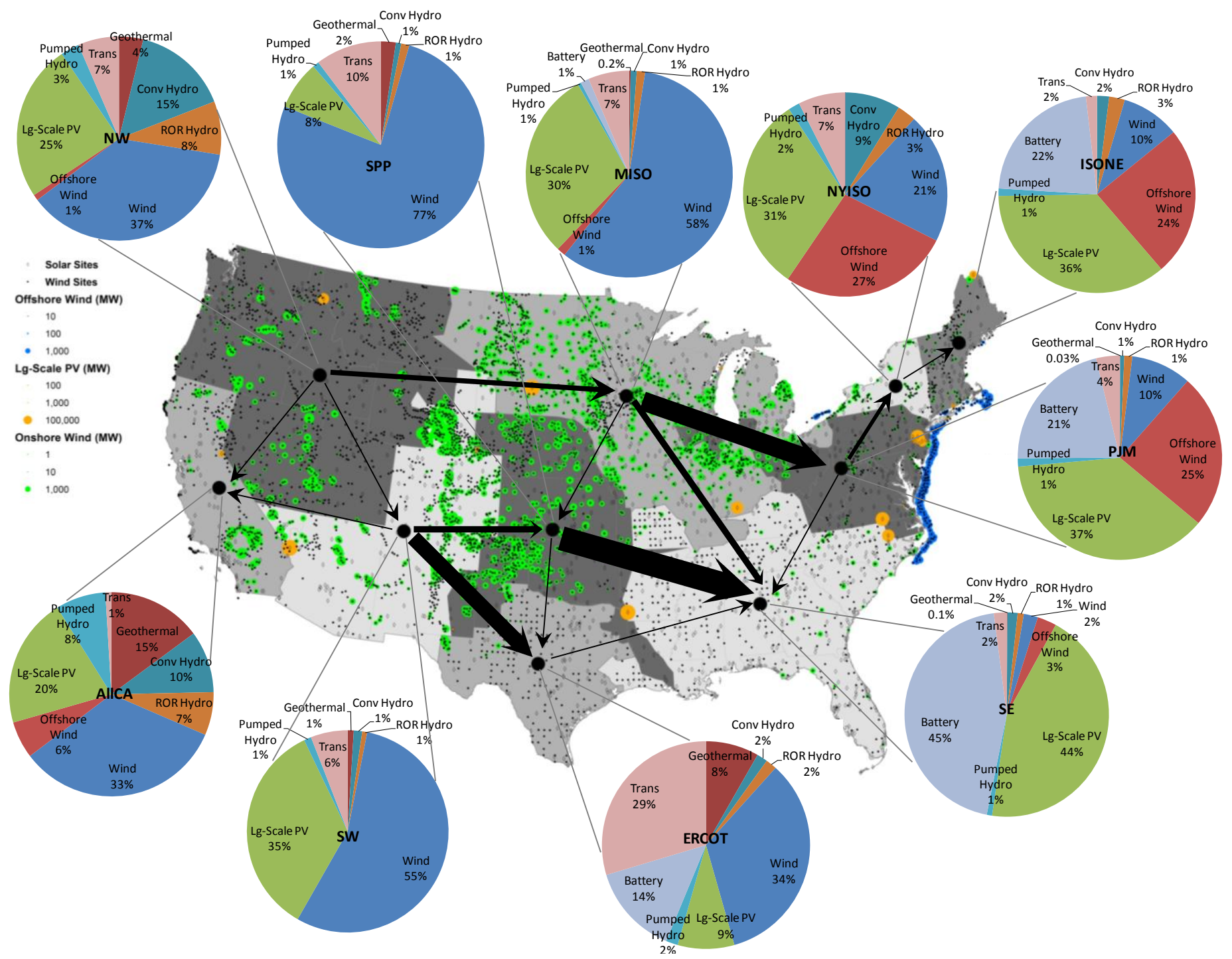

Figure 10 Breakdown of total annual system cost, wind and solar site buildouts, and net transmission flows for interconnected US with $100 \%$ aggregate RPS target. Pie charts show relative contribution of each built generator and storage technology to the total annual cost in each region. Arrow show dominant direction of transmission, and weight of line shows relative magnitude of total annual net flow, with the largest magnitude being 389TWh from SPP to SE. Actual achieved RPS in each region, as well as the aggregate RPS, is 100\%. Results are based on a representative subset of 14 days. 
Similar observations were made for the contiguous US system as for WECC. First, transmission occupied a small portion at roughly $5 \%$ of the total cost for both RPS targets. This was more than the WECC case and was primarily due to the need for enhanced east-west and ERCOT connections. Secondly, as observed in the WECC case, the interconnected system had less solar and storage deployment. Instead, the interconnected US system utilized more wind, especially in the Great Plains, Rocky Mountains, and Pacific Northwest (Figure 10). Third, based on these results for the US, which agree with those for the WECC spatial extent, enhancing the transmission system to access a greater diversity and quality of resources from larger geographic areas was more economically favorable than accessing only local resources with increased storage. In the independent regions case, storage coupled with large-scale PV was especially prevalent in the eastern half of the US.

Note that the large presence of storage, particularly battery storage, is also complemented by large transmission interchanges. These results do not single out transmission or storage as being superior to the other, but rather that both are needed in different combinations depending on the existing infrastructure, regional demand, and regional resource mix. The decision to build large quantities of storage in these cases reflects the need for greater system flexibility. In practice, this flexibility could come from other storage technologies, responsive demand, or new load sources. The impact of flexible load - in the form of flexible load from PEV charging with comparable scenarios in POWER - has been found to further shift the system's economic preference from a more regional basis with greater shares of relative storage to a more aggregated, interconnected system with more dispersed renewables and less relative local storage [6]. Future work should evaluate the impact of a larger suite of flexible resources.

In these contiguous US scenarios, renewable overgeneration became increasingly significant at higher RPS targets. About $6 \%$ of the total wind, solar, and hydroelectric generation was curtailed in the $80 \%$ RPS case and about $30 \%$ in the $100 \%$ RPS case. The National Renewable Energy Laboratory (NREL) Renewable Electricity Futures study, which solved for the costoptimal portfolio of generators, storage, and transmission using the ReEDS model, reflected similar results for an $80 \%$ renewable US system. This NREL study found that $8-10 \%$ of total solar, wind and hydroelectric generation was curtailed, almost all of which occurred in the western and eastern interconnects and varied by season [3].

Regional disparity was again seen in the level of renewable penetration for an $80 \%$ aggregate RPS target. For the interconnected US, the ISONE, PJM, and SE regions all achieved less than the $80 \%$ target $(57 \%, 56 \%$, and $70 \%$, respectively), while most other regions become fully renewable to compensate. As seen in the WECC 80\% RPS case, this disparity resulted in more natural gas generation in the sub-performing regions (ISONE, PJM, and SE) compared to the individual regions scenario.

The site buildout results shown here for the contiguous US also agree reasonably well with those from NREL's Renewable Electricity Futures Study [3]. In NREL's study, wind was heavily installed in the Great Plains, Great Lakes, Central, Northwest, and Mid-Atlantic areas (roughly corresponding to the MISO, SPP, NW and PJM regions here), and solar was heavily installed in California, the Southwest, Texas, and the South (roughly corresponding to AllCA, SW, ERCOT, and SE regions here). Transmission buildout in this NREL study was mainly east-west oriented, 
with key additions to/from ERCOT, SE, SW, SPP, and MISO regions. These results suggest an emphasis on transmitting wind energy from the Great Plains to large-load, adjacent regions. The US results from the POWER model (Figure 10) were similar for wind and transmission, but solar was more uniformly developed with POWER.

\subsection{Temporal and spatial resolution}

The temporal and spatial resolution tradeoffs were evaluated in AllCA for 50\% and 80\% RPS targets. Four temporal resolutions were considered: time steps of every 1, 2, 4, and 8 hours, each for a full-year temporal extent. The 1-hour ("hourly") resolution is based on a representative subset of 56 days due to computational limitations as previously discussed. See Section 3.1 for details on the model modifications for the coarser time resolutions of 2 , 4 , and 8 hours. Two spatial resolutions were evaluated for the buildout of wind and solar sites: uniform buildout (i.e., each site within the region has the same fractional development of the available resource) and individual site buildout (i.e., the development of each site is a separate decision variable).

Figure 11 summarizes the total annual system cost for each temporal and spatial resolution combination and the percent increase in cost relative to the most "ideal" achievable combination of individual sites at an hourly temporal resolution (open star). A sub-hourly temporal resolution is the ultimate goal (filled star), but sub-hourly data was not available for the entire contiguous US.

Figure 11 shows that, at a high RPS target (80\%, shown on right), a coarser temporal resolution yielded a lower total annual system cost for both uniform and individual site buildouts; the coarser the resolution, the lower the cost. At a medium RPS (50\%, shown on left), however, a coarser temporal resolution had comparatively little effect on the total cost. These differences are driven by the reduction in wind and solar variability due to averaging data within each time block. A less variable system (i.e., coarser temporal resolution) requires less flexibility and is therefore less expensive. This trend was more pronounced at a higher RPS target (80\%) where more wind and solar were deployed. At both RPS targets, the choice of spatial resolution had a more significant effect on total cost. The uniform buildout generally yielded a larger (suboptimal) total cost, especially in the high (80\%) RPS case with a 9\% increase in cost relative to the individual site buildout with an hourly temporal resolution. 

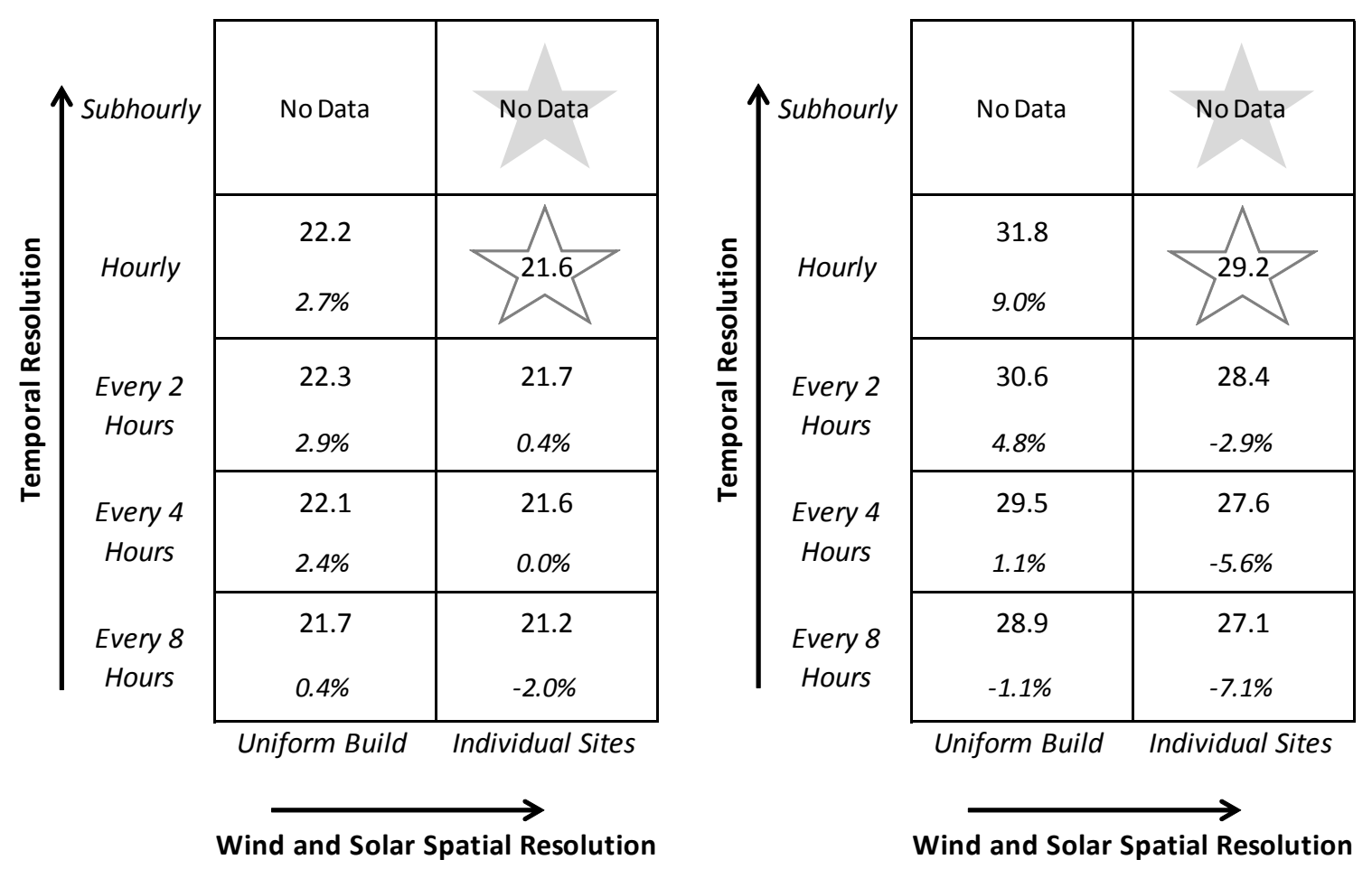

Figure 11 Temporal and spatial resolution cost tradeoffs for AllCA with 50\% and 80\% RPS targets. Total annual system cost (Billions of 2006USD/year) values are shown for each resolution combination, and the percent increases in cost are shown in italics relative to the most "ideal" achievable case of hourly individual sites (open star). The ultimate goal is to model individual wind and solar sites at a subhourly temporal resolution (filled star), but subhourly data was not available. Hourly values are based on a representative subset of 56 days, while coarser temporal runs are based on a full year of data.

\subsubsection{Spatial Resolution}

Figure 12 displays two different spatial resolutions for wind and solar site buildout with an hourly temporal resolution for AllCA at an 80\% RPS target. These maps reveal significant differences in wind and solar development between (1) considering each site independently for optimal site diversity, and (2) assuming an aggregated buildout across all sites uniformly as is done in many grid integration studies (e.g., [1]). The site-by-site buildout results (right side of Figure 12) found that optimal wind and solar development is clustered in southern California. However, since POWER assumes a "copper plate" within each region, the cost of transmitting the aggregated output from these sites to the coastal load centers is an important additional consideration that could alter these results. For example, results in Shawhan [35] indicate that that the spatial resolution of transmission-system detail in production cost models can impact greenhouse has emission results by as much as $20 \%$. 


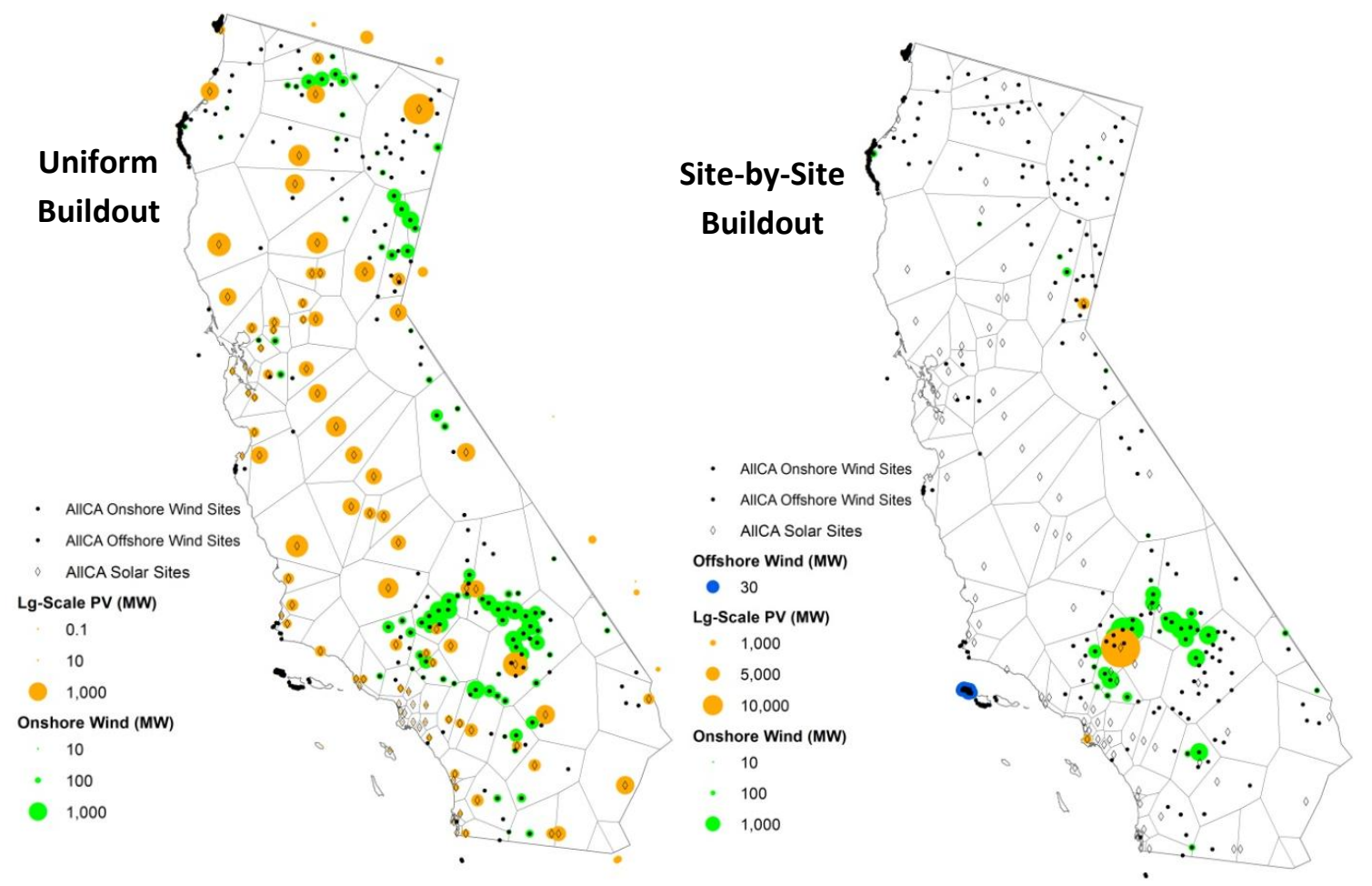

Figure 12 Wind and solar installed capacity by site for uniform and site-by-site buildout for AllCA at an $\mathbf{8 0 \%}$ RPS target. The size of the buildout circles corresponds to the installed capacity at each site (note that the circle sizes do not scale between the different generator types). All values are for cases with a representative subset of 56 days. No offshore wind was built in the uniform case (left). Results correspond to the "hourly" $80 \%$ RPS values in Figure 11.

A comparison of the breakdown of total annual costs between the two spatial resolution categories for AllCA at various RPS targets is shown in Figure 13. At high RPS targets, the difference in cost and renewable overgeneration become more pronounced between uniform buildout and site-by-site ("Indiv. Sites") buildout, with about a 10\% reduction in cost with siteby-site buildouts at a 100\% RPS target. These findings suggest that, depending on the application, the assumption to build wind and solar uniformly across all sites within a region may not be justified in studies focusing on high penetrations of renewable energy. 


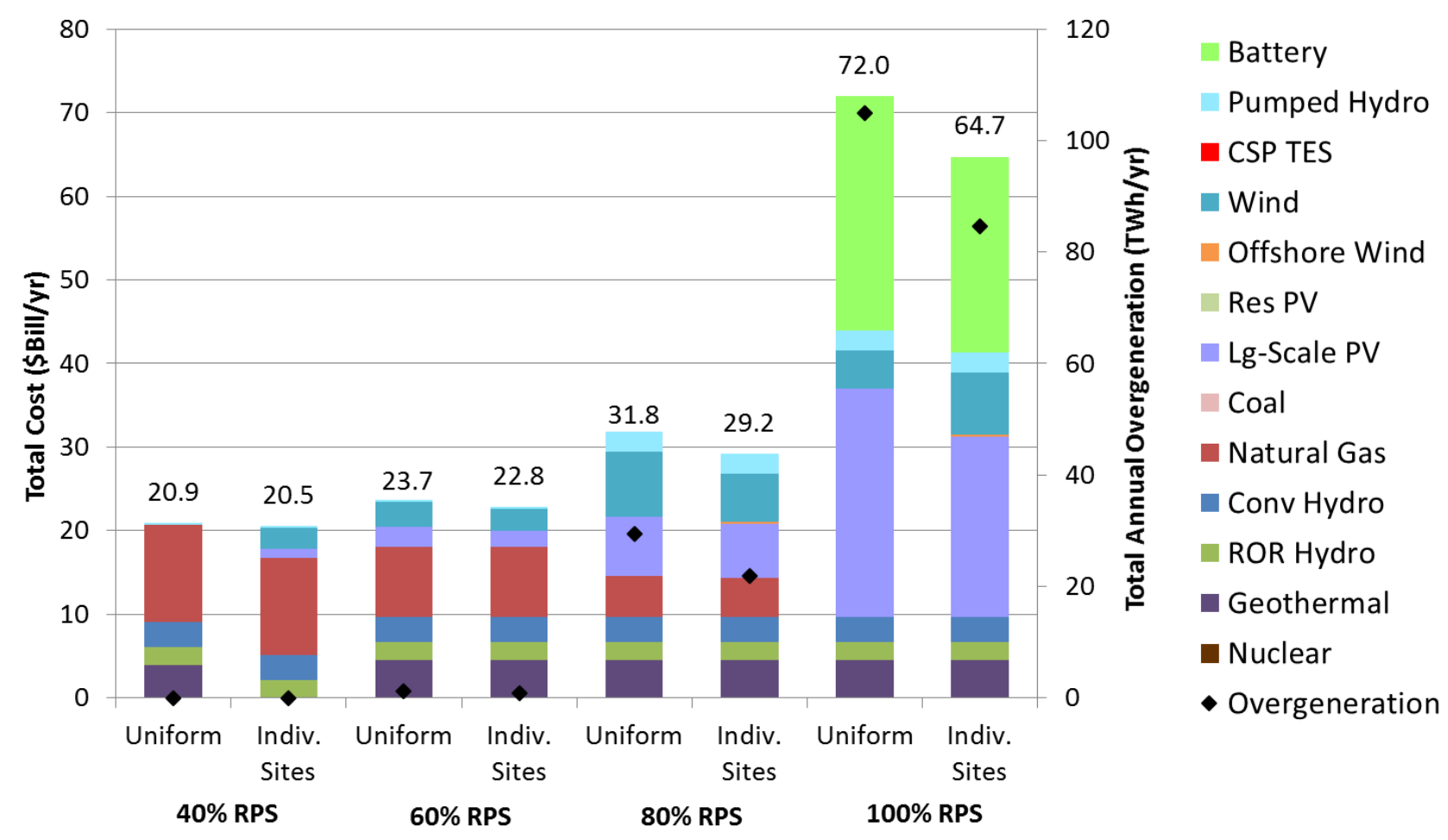

Figure 13 Total annual system cost and renewable overgeneration comparison for wind and solar uniform and site-by-site buildouts for AllCA at various RPS targets. All values are based on a representative subset of 56 days. Costs are in 2006USD.

\subsubsection{Temporal Resolution}

The breakdown of total annual costs across the four temporal resolution categories (1-, 2-, 4-, and 8-hour blocks) is shown in Figure 14 for AllCA at an 80\% RPS target. As observed in the resolution summary matrix for this RPS target (Figure 11), the total system cost decreases as the temporal resolution becomes coarser, with a difference in cost of about $10 \%$ between the hourly (least coarse) and 8-hour (most coarse) cases. Figure 14 reveals that the main driver of this trend is the contribution from wind and solar. Additionally, a coarser temporal resolution significantly underestimates the overgeneration of renewables. These results are due to the loss of intra-time block variability as previously discussed. The relatively large differences in cost and overgeneration and loss of variability with these reduced temporal resolution scenarios suggest that the alternative temporal reduction option - using a representative subset of days - provides a more accurate overall representation of the system components and should be the preferred method when computational limitations require a reduced temporal treatment. 

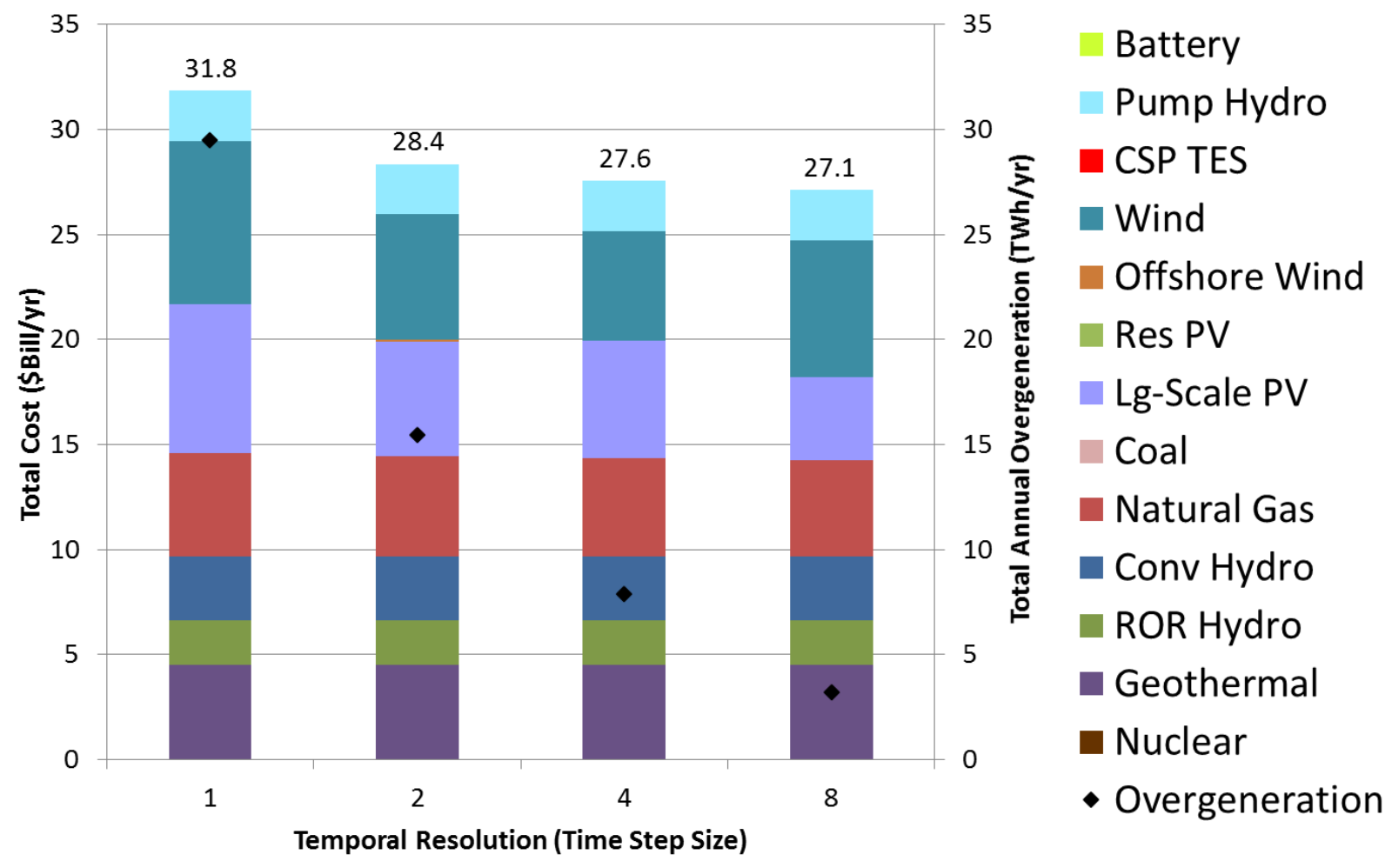

Figure 14 Total annual system cost and renewable overgeneration for various time step sizes (hours) for AllCA at an $80 \%$ RPS target. Costs are in 2006USD.

\section{Summary and conclusions}

This empirical study presents a link between various power system modeling choices and their computational and accuracy tradeoffs using the POWER least-cost optimization planning model for scenarios of a highly renewable US system. The first half of the study analyzed common temporal modeling simplifications, particularly the use of a representative subset of days within a full-year temporal extent. These days were selected as a combination of extreme event and typical days. Low accuracy losses of generally $\leq 6 \%$ across various representative day subset sizes with a reduced form model of POWER suggest that using a subset of days is justified by the associated computational savings. However, this accuracy result is likely dependent upon the model formulation and corresponding input assumptions, particularly with respect to the storage model formulation, cost assumptions, variable renewable curtailment treatment, and inclusion of multiple energy sectors. Across multiple scenarios, computational run time was found to increase quadratically with increasing subset size, while accuracy increased roughly logarithmically.

Because of the temporal disjointedness of most days in the representative subsets, the chronological tracking of storage was limited to the 24 hours within each representative day. Model outputs were found to be sensitive to the presence and/or choice of this daily balance constraint. While total system costs varied by $<2 \%$, the storage performance noticeably differed between a full chronological tracking of storage and the daily storage balance constraint. This highlights the need for proper temporal treatment and daily/weekly/seasonal storage balancing constraints in power system planning models, especially at high RPS targets when storage tends 
to play a greater role. Overall, these temporal modification findings suggest that using a representative subset of days with an associated daily storage balance provides a reasonable suboptimal approximation in POWER.

The second half of this study quantified cost tradeoffs of various temporal and spatial modeling adjustments. These adjustments resulted in small changes of generally $<15 \%$ in cost for the AllCA region, WECC, and contiguous US. Summary matrices of these tradeoffs can help model builders and users determine the most appropriate treatment of temporal and spatial components for their given application. The most significant difference in total cost was due to the effect of aggregation across increasing spatial extents, with about a $14 \%$ reduction in total cost for the WECC area at an $80 \%$ RPS target, and a $42 \%$ reduction for the contiguous US at a $100 \%$ RPS target. The impact of the spatial resolution of wind and solar site development (individual site buildout versus uniform development across all sites) also had large impacts on total system cost in the test case of the AllCA region. Compared to the uniform buildout case, the site-by-site buildout case had about a 10\% reduction in cost at a 100\% RPS target. These findings highlight the benefit of interconnecting large geographic areas and modeling individual wind and solar sites for optimal system planning in highly renewable energy futures. In addition, accuracy losses were about $10 \%$ with a coarser temporal resolution, indicating that the use of a representative subset of days is the preferred method when computational limitations require a reduced temporal treatment.

The findings here reflect technically feasible scenarios for a simplified contiguous US electricity system and ignore many social, environmental, and political barriers, which may slow or prevent actual implementation. The modeling tradeoff results presented here focused on capacity expansion modeling of the power system without consideration of new technologies, new loads from other sectors that are currently not electrified, other applications that could use otherwise curtailed energy, or responsive demand. The flexibility capabilities of these sources may impact the modeling tradeoff results presented here by, for example, re-defining "extreme days." Additionally, since POWER finds the least-cost portfolio and does not evaluate revenue streams, it cannot capture the full value for all of the system components. This includes arbitrage for storage and flexible demand, as well as revenue opportunities for generators in adjacent markets. Future work should examine the impacts of these additional factors in the tradeoffs between the various decision variables. Future work should also focus on establishing a more robust dayselection method or alternative model formulations for reducing model run time and memory requirements without sacrificing model accuracy.

\section{Acknowledgements}

BAF gratefully acknowledges financial support from a National Defense Science and Engineering Graduate (NDSEG) fellowship, a National Science Foundation (NSF) graduate research fellowship, and a Stanford University Charles H. Leavell Graduate Student Fellowship. The authors also thank Mary Cameron for valuable feedback on the draft of this paper. 


\section{References}

[1] C. Budischak, D. Sewell, H. Thomson, L. Mach, D.E. Veron, W. Kempton, Cost-minimized combinations of wind power, solar power and electrochemical storage, powering the grid up to 99.9\% of the time, J. Power Sources. 225 (2013) 60-74. doi:http://dx.doi.org/10.1016/j.jpowsour.2012.09.054.

[2] J. Nelson, J. Johnston, A. Mileva, M. Fripp, I. Hoffman, A. Petros-Good, et al., High-resolution modeling of the western North American power system demonstrates low-cost and low-carbon futures, Energy Policy. 43 (2012) 436-447. doi:10.1016/j.enpol.2012.01.031.

[3] National Renewable Energy Laboratory, Renewable Electricity Futures Study, Golden, CO, US, 2012. http://www.nrel.gov/analysis/re_futures/.

[4] G.J. Blanford, J.H. Merrick, D. Young, A Clean Energy Standard Analysis with the US-REGEN Model, Energy J. 35 (2014). doi:http://dx.doi.org/10.5547/01956574.35.SI1.8.

[5] A.E. MacDonald, C.T.M. Clack, A. Alexander, A. Dunbar, J. Wilczak, Y. Xie, Future costcompetitive electricity systems and their impact on US CO2 emissions, Nat. Clim. Chang. 6 (2016) 526-531. doi:10.1038/nclimate2921.

[6] B.A. Frew, S. Becker, M. Dvorak, G.B. Andresen, M.Z. Jacobson, Flexibility Mechanisms and Pathways to a Highly Renewable U.S. Electricity Future, Energy. 101 (2016) 65-78. doi:10.1016/j.energy.2016.01.079.

[7] B.A. Frew, Optimizing the Integration of Renewable Energy in the United States, Ph.D. Dissertation, Stanford University, 2014. http://purl.stanford.edu/hr320qr0229.

[8] P. Sullivan, K. Eurek, R. Margolis, Advanced Methods for Incorporating Solar Energy Technologies into Electric Sector Capacity-expansion Models: Literature Review and Analysis, National Renewable Energy Laboratory, NREL/TP-6A20-61185, Golden, CO, 2014.

[9] W. Short, P. Sullivan, T. Mai, M. Mowers, C. Uriarte, N. Blair, et al., Regional Energy Deployment System (ReEDS), National Renewable Energy Laboratory, NREL/TP-6A20-46534, Golden, CO, US, 2011. http://www.nrel.gov/analysis/reeds/pdfs/reeds_documentation.pdf.

[10] M. Fripp, Switch: A Planning Tool for Power Systems with Large Shares of Intermittent Renewable Energy, Environ. Sci. Technol. 46 (2012) 6371-6378. doi:10.1021/es204645c.

[11] Electric Power Research Institute, PRISM 2.0: Regional Energy and Economic Model Development and Initial Application, Palo Alto, CA, 2013. http://www.epri.com/abstracts/Pages/ProductAbstract.aspx?ProductId=000000003002000128.

[12] U.S. Energy Information Administration, The Electricity Market Module of the National Energy Modeling System: Model Documentation Report, DOE/EIA-M068(2011), 2011. ftp://ftp.eia.doe.gov/modeldoc/m068(2011).pdf. 
[13] R. Alliss, D. Apling, M. Mason, H. Kiley, G. Higgins, Applications of the Renewable Energy Network Optimization Tool (ReNOT) for use by Wind \& Solar Developers: Part II, 91st Am. Meteorol. Soc. Annu. Meet. (2011).

[14] R. Alliss, D. Apling, M. Mason, H. Kiley, K. Darmenova, G. Higgins, Introducing the Renewable Energy Network Optimization Tool (ReNOT): Part I, 91st Am. Meteorol. Soc. Annu. Meet. (2011).

[15] T. Mai, C. Barrows, A. Lopez, E. Hale, M. Dyson, K. Eurek, Implications of Model Structure and Detail for Utility Planning: Scenario Case Studies Using the Resource Planning Model, National Renewable Energy Laboratory, NREL/TP-6A20-63972, Golden, CO, US, 2015.

[16] C. Barrows, T. Mai, E. Hale, A. Lopez, K. Eurek, Expansion, Considering Renewables in Capacity Dispatch, Models: Capturing Flexibility with Hourly, in: IEEE Power Energy Soc. Gen. Meet., Denver, 2015.

[17] P. Nahmmacher, E. Schmid, L. Hirth, B. Knopf, Carpe Diem: A Novel Approach to Select Representative Days for Long-Term Power System Models with High Shares of Renewable Energy Sources, 2014. doi:10.2139/ssrn.2537072.

[18] B.A. Corcoran (Frew), N. Jenkins, M.Z. Jacobson, Effects of aggregating electric load in the United States, Energy Policy. 46 (2012) 399-416. doi:10.1016/j.enpol.2012.03.079.

[19] M.Z. Jacobson, M.A. Delucchi, M.A. Cameron, B.A. Frew, A Low-Cost Solution to the Grid Reliability Problem With 100\% Penetration of Intermittent Wind, Water, and Solar for all Purposes, Proc. Natl. Acad. Sci. 112 (2015). doi:10.1073/pnas.1510028112, 2015.

[20] D. Connolly, H. Lund, B.V. Mathiesen, Smart Energy Europe: The technical and economic impact of one potential 100\% renewable energy scenario for the European Union, Renew. Sustain. Energy Rev. 60 (2016) 1634-1653. doi:10.1016/j.rser.2016.02.025.

[21] J. Merrick, Modeling the Economics of Variable Electric Generation Resources, in: INFORMS, Phoenix AZ, 2012.

[22] R. Green, N. Vasilakos, Divide and Conquer? k-Means Clustering of Demand Data Allows Rapid and Accurate Simulations of the British Electricity System, IEEE Trans. Eng. Manag. (2014) 110 .

[23] C.H. Marton, A. Elkamel, T.A. Duever, An order-specific clustering algorithm for the determination of representative demand curves, Comput. Chem. Eng. 32 (2008) 1373-1380.

[24] P.G. Brodrick, C.A. Kang, A.R. Brandt, L.J. Durlofsky, Optimization of CCS-Enabled Coal-GasSolar Power Generation, Energy. (2015).

[25] E.K. Hart, Optimization-Based Modeling Methods for Reliable Low Carbon Electricity Portfolios, Ph.D. Dissertation, Stanford University, 2012. http://purl.stanford.edu/hj267zv4525.

[26] A. Schrijver, Theory of Linear and Integer Programming, John Wiley \& Sons, Ltd, West Sussex, England, 1986.

[27] V. Klee, G.J. Minty, How good is the simplex algorithm?, in: Shisha, Oved. Inequalities III (Proceedings Third Symp. Inequalities, Sept. 1-9, 1969, New York-London: Academic Press, University of California, Los Angeles, Calif., 1972: pp. 159-175.

[28] M. Nicolosi, The Importance of High Temporal Resolution in Modeling Renewable Energy Penetration Scenarios, in: 9th Conf. Appl. Infrastruct. Res. TU Berlin, Berlin, Ger. Oct. 8-9, 2010, LBNL Paper LBNL-4197E, 2011. 
[29] R. Kannan, H. Turton, A Long-Term Electricity Dispatch Model with the TIMES Framework, Environ. Model. Assess. 18 (2012) 325-343. doi:10.1007/s10666-012-9346-y.

[30] J. King, B. Kirby, M. Milligan, S. Beuning, Flexibility Reserve Reductions from an Energy Imbalance Market with High Levels of Wind Energy in the Western Interconnection, National Renewable Energy Laboratory NREL/TP-5500-52330, Golden, CO, US, 2011.

[31] J.F. DeCarolis, D.W. Keith, The economics of large-scale wind power in a carbon constrained world, Energy Policy. 34 (2006) 395-410. doi:10.1016/j.enpol.2004.06.007.

[32] K. Schaber, F. Steinke, P. Mühlich, T. Hamacher, Parametric study of variable renewable energy integration in Europe: Advantages and costs of transmission grid extensions, Energy Policy. 42 (2012) 498-508. doi:http://dx.doi.org/10.1016/j.enpol.2011.12.016.

[33] T.W. Nelson, Bath County Power Station Manager, (2012).

[34] A. Mills, R. Wiser, Implications of wide-area geographic diversity for short-term variability of solar power, Lawrence Berkeley National Laboratory LBNL-3884E, 2010.

[35] D.L. Shawhan, J.T. Taber, D. Shi, R.D. Zimmerman, J. Yan, C.M. Marquet, et al., Does a detailed model of the electricity grid matter? Estimating the impacts of the Regional Greenhouse Gas Initiative, Resour. Energy Econ. 36 (2014) 191-207. doi:10.1016/j.reseneeco.2013.11.015. 